\title{
Bioactive Microbial Metabolites
}

\section{A Personal View}

János Bérdy
Received March 4, 2004 / Accepted September 28, 2004

(C) Japan Antibiotics Research Association
Abstract The short history, specific features and future prospects of research of microbial metabolites, including antibiotics and other bioactive metabolites, are summarized. The microbial origin, diversity of producing species, functions and various bioactivities of metabolites, unique features of their chemical structures are discussed, mainly on the basis of statistical data. The possible numbers of metabolites may be discovered in the future, the problems of dereplication of newly isolated compounds as well as the new trends and prospects of the research are also discussed.

Keywords bioactive microbial metabolites, biological origin, antibiotic and other bioactivities, chemical features, dereplication, the number of known compounds, future chances

\section{Contents}

\section{Introduction}

Natural Products, Secondary Microbial Metabolites, Antibiotics

Short History of the Antibiotic Research

Definitions

Specific Features of Microbial Metabolites:

Origin-The Producers of Microbial Metabolites (Taxonomic diversity)

Distribution of Bioactive Natural Products

The Research of Microbial Metabolites (Statistical evaluations)
Biological Activity (Biodiversity)

Function of Secondary Metabolites (Biological functionality)

Bioactivities of Secondary Metabolites

Main Types of Activities (Statistical evaluations)

Chemical Structures (Chemical diversity)

Increasing the Biosynthetic Diversity

Structural Features of Microbial Metabolites (Statistical evaluations)

The Number of Microbial Metabolites-How many microbial metabolites are known, indeed?

Problems of the Dereplication and the Nomenclature

Hidden Identities

Obscure origin of Compounds

Conclusions and Prospects-How many microbial metabolites may be discovered in the future?

Future Chances (Diversifying of microbial products)

New Producers

Screening Protocols

Data Management and Cooperation

Epilogue

\section{Introduction}

The story of antibiotics had started more than half a century ago. My first personal meeting with these fascinating compounds was going back to more than forty years. From that time I devoted a significant part of my life 
to these exciting and fantastic compounds. Due to the interdisciplinary nature of this area, being originally organic chemist, it was a great experience to obtain every possible knowledge of the related disciplines. In the first years of my career I had been working in various antibiotic screening projects, and then I had worked almost exclusively in the field of data management, collecting the diverse data of bioactive natural compounds.

In the present paper, similarly to my previous reviews, I try to summarize my ideas and imaginations about the past, present and future of the area of antibiotics research or in wider sense, the story and main trends of the discovery and the important characteristics of the bioactive microbial metabolites [1 9].

Their most important specific features; the biological origin, bioactivities, chemical structures, possible numbers and in the light of recently obtained statistical data, some interesting recent trends and the future prospects of these compounds, playing an important role in the area of natural products research, will be discussed.

\section{Methods}

The numbers of known compounds, producers and bioactivities in this review are estimated mainly on the basis of detailed literature survey and on study of various databases. In this study most data are derived from my near to thirty years old, but continuously updated database (BNPD) which attempt to cover all bioactive microbial metabolites and, as far as possible, the whole scientific and patent literature, covering near to one million references $[3,10]$. Over $90 \%$ of the compounds in this database have known chemical structure or they are chemically exactly characterized. Several conclusions and calculations are based on the data derived from other databases [11,12], handbooks and reviews [13 24].

I apologize for the huge mass of statistical data which will be covered in the followings, but as several of them have never been published in the past, I hope, it may be interesting for some people in the scientific community.

Natural Products, Secondary Microbial Metabolites, Antibiotics

\section{Short History of the Antibiotic Research}

The antibiotic research from the discovery of Fleming to our days has been a fascinating, exciting, continuously changing and developing adventure. As a result of the frenzied research of the past more than 50 years, in our days ten thousands of natural products derived from microbial sources are known. Interest towards the field were generally increasing, although sometimes declining, interest and the whole story shows some cyclic features with successes and failures and evolved around changing clinical needs and new enabling technology.

After the revolution in the "heroic" or "golden era", in the forties and early fifties, when almost all groups of important antibacterial antibiotics (tetracyclines, cephalosporins, aminoglycosides, macrolides) were discovered, the success story had continued. It seemed in the fifties and sixties that the main problems of chemotherapy had been solved. Antibiotics discovered in this period were mainly isolated from Streptomyces species, representing some 70 to $80 \%$ of the all isolated compounds. They were primarily active against bacteria and fungi. In this period the discovery of antitumor, antiviral and non antibiotic-enzyme inhibitory-metabolites, had just started.

In the next period, between the seventies and nineties the efficiency of research had decreased. The costs of research had increased, and although the number of discovered new compounds still increased, they were mainly analogues of known compounds. The scope of search for various bioactive microbial products had, however, broadened. The exploration and wide utilization of the antitumor (doxorubicin) and agricultural antibiotics, (antiparasitic avermectin, feed additive monensin and herbicide glufosinate), the early discoveries of utilization of microbial metabolites in the pharmacological fields (cyclosporin, statins), were important new features. The problems of chemotherapy (emerging new pathogens and escalation of multi-resistant strains) had become serious. In this period, besides the leading role of actinomycetes products $(65 \sim 70 \%)$, the considerable increase of the discovery of "rare actinomycetes" products (up to 30\%) were also noticeable. The chemical structures of almost all discovered compounds had been elucidated.

After these years, from the nineties, the exponential increase of the number of new metabolites (mainly nonantibiotic compounds, analogous and minor compounds), still continued but the occurrence of new chemical types had diminished. Due to urgent clinical needs, the increasing serious problems of chemotherapy (multi-resistant strains, reappearing mycobacteria, HIV, etc.), new challenges in the therapy of physiological diseases and in the agriculture, the renovation of the classical screening methods, allowed by the new technologies, were highly required. Methodological innovation accompanied by changes in conception. The cost effective high throughput screening (HTS) methods (robotics, instrumentation) based on mainly 
biochemical rationale and the wide application of diverse genetic manipulations, became more and more widespread. The rapid progress of the human genome project provided access to a wide range of new molecular targets implicated in diverse non infectious diseases. The screenings have become more efficient than ever. The dramatic increase of the isolation of non-antibiotic compounds with pharmacological and agricultural activities, (up to $60 \sim 65 \%$ of all isolated compounds), the increasing share of various fungal metabolites (up to 50\%), and the chemical synthesis of more and more complicated structures, are the most characteristic features of this present period. It seems, with the opening of the 21 st Century, a new era of antibiotic research had opened.

Notwithstanding the failures, the almost exponential increasing of the total number of discovered compounds in the last decades surprisingly became constant. In 1940 only $10 \sim 20$, in $1950300 \sim 400$, in 1960 approximately 800 1000 and in 1970 already 2500 antibiotics were known. From that time the total number of known bioactive microbial metabolites has doubled in every ten years. In 1980 about 5000, in 199010000 and in 2000 already almost 20000 antibiotic compounds were known. By the end of 2002 over 22000 bioactive secondary metabolites (including antibiotics) were published in the scientific and patent literature. On Fig. 1 the total number of discovered antibiotics is summarized chronologically from 1950 to 2000 in five years period intervals, indicating their main sources, such as Streptomyces, rare actinomycetes, fungi and bacteria (1a), as well as their percentages indicated in the same periods $(1 \mathrm{~b})$.

Unfortunately, these quantitative improvements do not mean similar qualitative, practical results. The expected corresponding spectacular successes, in spite of the great scientific and technical developments, are still waiting to be fulfilled.

Because of the fascinating technical improvements in the separation and isolation techniques, in our days it is likely that-over the antibiotics-close to one million naturally occurring compounds are known, however, it is supposed, that today (at the end of 2003) due to the intensive use of genetic methods and high throughput screening techniques (HTS), the number of existing and detected compounds may be definitely higher. The majority of natural products is derived-besides the microbial products isolated from prokaryotic bacteria and eukaryotic microorganisms (protists), where almost all of the antibiotic producing microbes (except the animal protozoa) are belonging to-from higher plants and various animal organisms. Higher plant metabolites represent at least 500000 to 600000 compounds, covering a great number of common plant compounds, such as alkaloids, flavonoids, terpenoids, steroids, carbohydrates etc.

The natural products may exhibit various effects or are without any discovered interaction with other living organisms. In other words, they may show some kind of biological activity. The activity may be highly specific, exhibiting usually in low concentration (representing the usual bioactivities), or may be very unspecific (toxic) action. The exact number of bioactive natural products (compounds with discovered bioactivity or toxicity), is almost undeterminable, but this figure by all means is at least 200000 to 250000 , - including more than 20000 microbial metabolites.

The approximate number of the known natural products derived from the main types of Plant and Animal organisms, is summarized in Table 1.

\section{Definitions}

The antibiotics and similar compounds form perhaps the

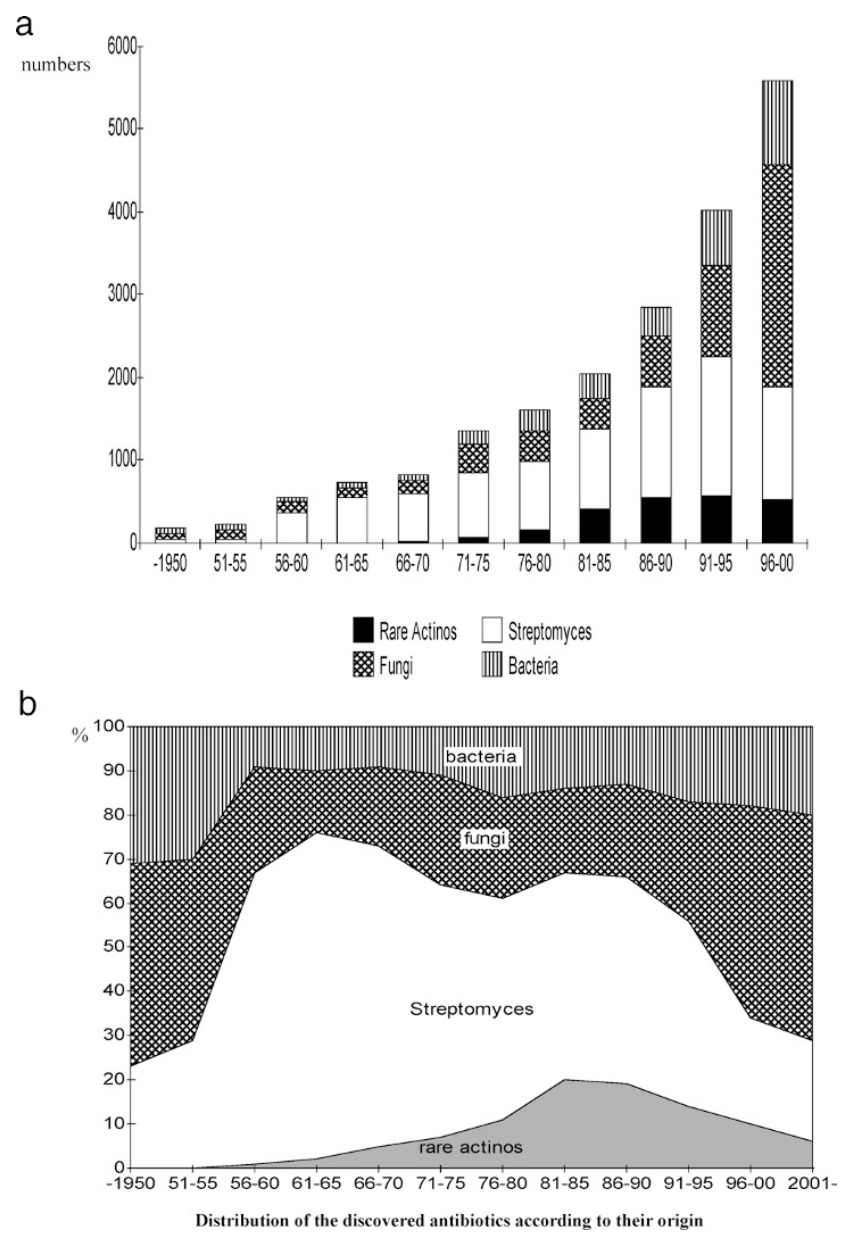

Fig. 1 Distribution of the discovered antibiotics according to their origin. 
Table 1 Approximate number of known natural products

\begin{tabular}{|c|c|c|c|}
\hline Source & All known compounds & "Bioactives" & Antibiotics \\
\hline Natural Products & over one million & 200000 to 250000 & 25000 to 30000 \\
\hline Plant Kingdom & 600000 to 700000 & 150000 to 200000 & $\sim 25000$ \\
\hline Microbes & over 50000 & 22000 to 23000 & $\sim 17000$ \\
\hline Algae, Lichens & 3000 to 5000 & 1500 to 2000 & $\sim 1000$ \\
\hline Higher Plants & 500000 to 600000 & $\sim 100000$ & 10000 to 12000 \\
\hline Animal Kingdom & 300000 to 400000 & 50000 to 100000 & $\sim 5000$ \\
\hline Protozoa & several hundreds & 100 to 200 & $\sim 50$ \\
\hline Invertebrates: & $\sim 100000$ & $?$ & $\sim 500$ \\
\hline Marine animals & 20000 to 25000 & 7000 to 8000 & 3000 to 4000 \\
\hline Insects/worms/etc. & 8000 to 10000 & 800 to 1000 & 150 to 200 \\
\hline $\begin{array}{l}\text { Vertebrates (mammals, fishes, } \\
\text { amphibians, etc.) }\end{array}$ & 200000 to 250000 & 50000 to 70000 & $\sim 1000$ \\
\hline
\end{tabular}

most interesting, most dynamically increasing, and one of the practically most important group of the natural products.

The usual definition of natural products in the widest sense emphasizes that they "are chemical (carbon) compounds isolated from diverse living things". These compounds may derive by primary or rather secondary metabolism of living organisms. The primary metabolites (polysaccharides, proteides, nucleic and fatty acids) are common in all biological systems. The secondary metabolites are, however, low molecular (MW<3000), chemically and taxonomically extremely diverse compounds with obscure function, characteristic mainly to some specific, distinct types of organisms.

An important part of the natural products, the group of small molecular secondary metabolites of microorganisms, usually exhibits some kinds of biological activities, and these compounds, the bioactive secondary microbial metabolites, represent the main topic of the recent review.

The exact definition and the real position and function/s of secondary metabolites for long time is the most disputed and most obscure field in the whole area of microbiology. They seem to be absolutely needless for the producers, having no any apparent function in their life cycle. Most characteristic features are their incredible array of unique chemical structures and their very frequent occurrence and versatile bioactivities [25].

A few words have to be say about the term "bioactivity" or "biological activity". This activity may appear at the in vitro molecular level or may be studied at the in vivo level, the activity on the whole organism. Taking into consideration the possible differences and specific features surely manifesting in these different levels, in the following this term will be used generally for any types of interactions between chemicals and any kinds of molecular targets or living organisms.

Secondary metabolites are known from the ancient times, and they were mainly botanicals. The first crystalline fungal product from Penicillium glaucoma considered as microbial secondary metabolite was mycophenolic acid, discovered in 1896 by Gosio.

The secondary metabolites isolated from microbes and exhibits either antimicrobial (antibacterial, antifungal, antiprotozoal), antitumor and/or antiviral activities, used to be called as antibiotics. In the light of our recent knowledge, however, the term "antibiotic" is more or less an outworn conception, but a similar, simple, expressive, inclusive term is still waiting to be invented. We use it because we have not found better one. Today its original definition actually should be extended to all of those (microbial) secondary metabolites which regulates growth processes, replications, and/or exhibits some kind of responding (regulating, inhibiting, stimulating) action to the (life cycle of) prokaryotic or eukaryotic cells at the biochemical level, in minimal concentration. This broadest definition should cover besides the so-called "classical antibiotics" (microbial compounds exhibiting antimicrobial and/or antitumor and/or antiviral activities) practically all bioactive compounds obtained either from microbes or from any other living things. In the followings, the term antibiotic/s sometimes used, in a logical sense, instead of the term bioactive microbial metabolite/s. Further on, I try to call consistently the non-antibiotic bioactive compounds exhibiting exclusively some kind of other biological activities as "other bioactive" metabolites/compounds. It is possible to name all of these compounds, however, somewhat more correctly but less inclusively, by the terms as (natural) bioregulators or biochemical modulators. The 
problem of correct definition is still open and the borderlines between the overlapping bioactive compounds derived from various sources naturally are still far from being clear. No doubt that the "classical" antibiotics form only a part (perhaps a smaller part) of the possible bioactive metabolites of microorganisms. They represent only the top of the iceberg, the deep still only touched. The reason for the present existence of that relatively great number of antibiotics, is undoubtedly based on their easy detection and because for a long time only these compounds had been looked for. Their huge and quick success is simply derived from the fact that in the fifties they just had fulfilled a great demand in the chemotherapy. It was a never returning opportunity that led to the wonderful "golden era" of the antibiotics research.

The practical importance of antibiotics and other secondary metabolites is tremendous. They are widely used in the human therapy, veterinary, agriculture, scientific research and in countless other areas.

In general, natural products including the microbial metabolites may be practically utilized in three different ways:

- Applying the natural/fermentation product directly in the medicine, agriculture, or in any other fields;

- Using as starting material for subsequent chemical or microbiological modification (derivatization);

- They can be used as lead compounds for chemical synthesis of new analogs or as templates in the rational drug design (RDD) studies.

In the following only the first aspect, the direct significance of the microbial compounds, will be discussed.

\section{Specific Features of Microbial Metabolites}

The most important, inherent characteristics of the bioactive microbial metabolites are their microbial origin, that is to say their specific microbial producers; their interaction with the environment, namely their various biological activities and last but not least their unique chemical structures.

\section{Origin-The Producers of Microbial Metabolites (Taxonomic Diversity)}

\section{Distribution of Bioactive Natural Products}

Antibiotics and similar natural products, being secondary metabolites can be produced by almost all types of living things. They are produced by prokaryotic (Prokaryotae, Monera) and eukaryotic organisms belonging to the Plant and Animal Kingdom, alike. The secondary metabolite producing ability, however, is very uneven in the species of living world. In the Prokaryotae and Plant Kingdom there are distinct groups of organisms, namely unicellular bacteria, eukaryotic fungi and first of all filamentous actinomyces being the most frequent and most versatile producers.

In the group of prokaryotic, unicellular bacteria the Bacillus and Pseudomonas species are the most frequent producers. In the recent years Myxo- and Cyanobacteria species seem to join to these distinguished organisms as prolific species. Mycobacteria, mycoplasmatales and spirotheces are far less frequent producers. The total number of known bioactive compounds in this group is about $3800 ; 17 \%$ of all microbial metabolites. The filamentous actinomycetales species produces over 10000 bioactive compounds, 7600 derived from Streptomyces and 2500 from the so called rare actinomycetes (rare actino) species, represent the largest group (45\%) of bioactive microbial metabolites.

Among the eukaryotic microscopic fungi the producing capability of imperfect fungi, the ascomycetes and several other filamentous and endophytic fungal species are the most significant. The basidiomycetes are also frequently reported producers, while yeasts, phycomycetes, slime moulds rarely produce bioactive metabolites. The total number of bioactive fungal product is approximately 8600 , representing $38 \%$ of all microbial products.

From the known (altogether 22500) antibiotics and similar bioactive microbial compounds, less than one percent, only about 150 compounds, are in direct use in the human and veterinary medicine, and agriculture. In the human therapy about one hundred compounds, most of them derived from actinomycetales species, are in direct practical use.

As a best approximate, the total number of additional "inactive" microbial products is about 20000 to 25000 , therefore today close to 50000 microbial metabolites may be known.

According to the main types of microbial producers, the numbers of compounds, including both antibiotics, "other bioactive" metabolites, practically used compounds and the approximate numbers of the inactive microbial metabolites are summarized in the Table 2.

Over the bioactive microbial compounds from higher species of the Plants Kingdom such as algae, lichens and mainly from vascular plants, more than 13000 antimicrobial antitumor/antiviral compounds were isolated. Additionally, from species of the Animal Kingdom some 7000 bioactive compounds, derived from various marine and terrestrial animals, are also described (Table 3). 
Table 2 Approximate number of bioactive microbial natural products (2002). According to their producers

\begin{tabular}{|c|c|c|c|c|c|}
\hline Source & Antibiotics & $\begin{array}{c}\text { "Other bioactive" } \\
\text { metabolites }\end{array}$ & $\begin{array}{c}\text { Total bioactive } \\
\text { metabolites }\end{array}$ & $\begin{array}{c}\text { Practically used } \\
\text { (in human therapy) }\end{array}$ & $\begin{array}{c}\text { Inactive } \\
\text { metabolites }\end{array}$ \\
\hline Bacteria & 2900 & 900 & 3800 & $10 \sim 12(8 \sim 10)$ & 3000 to 5000 \\
\hline Atinomycetales & 8700 & 1400 & 10100 & $100 \sim 120(70 \sim 75)$ & 5000 to 10000 \\
\hline Fungi & 4900 & 3700 & 8600 & $30 \sim 35(13 \sim 15)$ & 2000 to 15000 \\
\hline Total & 16500 & 6000 & 22500 & $140 \sim 160(\sim 100)$ & 20000 to 25000 \\
\hline
\end{tabular}

Table 3 Approximate numbers of bioactive natural products (2002). Derived from higher forms of life

\begin{tabular}{|c|c|c|c|c|}
\hline Source & Antibiotics & "Other bioactive" & Total & \\
\hline Lichens & 160 & $?$ & 160 & \multirow{5}{*}{$\begin{array}{l}\text { Plant products } \\
13210\end{array}$} \\
\hline Algae & 620 & 730 & 1350 & \\
\hline$x^{2}$ & (non & (n) & (n) & \\
\hline Lower Plants (Bryophyta)* & 200 & $?$ & 200 & \\
\hline Higher Plants* & 11500 & $?$ & 11500 & \\
\hline Invertebrates* & 480 & $?$ & 480 & \multirow{15}{*}{$\begin{array}{l}\text { Animal products } \\
7280\end{array}$} \\
\hline Insects* & 320 & & 320 & \\
\hline Worms, others (crustaceans)* & 160 & & 160 & \\
\hline Marine Animals (total) & 3400 & 2700 & 6100 & \\
\hline Sponges & 1850 & 1500 & 3350 & \\
\hline Coelenterates & 630 & 570 & 1100 & \\
\hline Tunicates & 420 & 230 & 650 & \\
\hline Molluscs & 300 & 350 & 650 & \\
\hline Echinoderms & 220 & 60 & 280 & \\
\hline Bryozoa & 70 & 50 & 120 & \\
\hline Fishes* & 50 & $?$ & 50 & \\
\hline Amphibians, reptils* & 240 & $?$ & 240 & \\
\hline Terrestrial vertebrates* (total) & 410 & $?$ & 410 & \\
\hline Mammals* & 340 & & 340 & \\
\hline \multirow[t]{2}{*}{ Others (birds, etc.) ${ }^{*}$} & 50 & & 50 & \\
\hline & 16985 & & 20540 & \\
\hline
\end{tabular}

* Only antibiotic compounds, but no "other bioactive" compounds are covered.

? Difficult to estimate.

Besides the above microbes various microscopic algae, including seaweeds, dinoflagellates, diatoms (Chlorophyta, Rhodophyta, Phaeophyta, Ciliophora, etc. species) are relatively frequent producers with the more than 1300 bioactive metabolites produced by them. Lichens, and lower plants such as Bryophyta species, (liverworts, ferns and mosses) are also produce several hundreds of antibiotically active metabolites.

Higher green plants (Spermatophyta), gymnosperms and angiosperms alike are able to produce structurally unique secondary metabolites exhibiting antimicrobial and/or antitumor activities, covering close to 12000 "antibiotic" compounds. Of course, the common plant metabolites (alkaloids, flavonoids, terpenoids, etc.), which frequently exhibit various toxic and pharmacological effects, exhibiting some antimicrobial and antitumor/antiviral activities, are also included. In the widest sense all of the above compounds may also be considered as antibiotics.

In the Animal Kingdom in the last decades, the soft bodied invertebrated marine animals, belonging to the classes of Porifera, Mollusca, Cnidaria, Anthozoa, Echinodermata, and Bryozoa (sponges, molluscs, 
coelenterates, tunicates, echinoderms, etc.) proved to be rich sources of various bioactive metabolites. In 1972 only 25, in 1982 about 300, in 1992 approximately 1500 and in our days more than 6000 such marine derived bioactive compounds are known. Sponges alone produce more than 3300 antibiotics and other bioactive compounds. In the group of marine products, including the seaweeds, the nonantibiotic "other" bioactive compounds, constitute about half of the all presently known bioactive marine products. Noteworthy that these isolated "animal" compounds very frequently show surprising analogy to microbial or algal products. It is not surprising that in numerous occasions the active compounds isolated from these animals proved to be derived from the microorganisms living symbiotic with their host or maybe of dietary origin (see later). This problem is presently under detailed worldwide investigation.

Antimicrobially active compounds were also isolated from insects, worms, amphibians, and from various terrestrial vertebrates, alike. From animal microbes (protozoa, ciliates) only very few compounds were isolated possessing antibiotic activity. Antibiotic compounds are lacking or very rare among compounds derived from crustaceans, arachnids, fishes and birds.

The distribution of the antibiotic and other bioactive compounds derived from the higher forms of life is summarized on the Table 3.

The total number of bioactive secondary metabolites isolated from the higher forms of life is more than 20000 including close to 17000 antibiotic compounds, excluding the non-antibiotic higher plant and terrestrial animal products (whose numbers are difficult to estimate and in Table 3 are indicated by question-mark). In summary, today over 43000 bioactive natural products are known. This number includes antimicrobial, antitumor, antiviral and other bioactive microbial metabolites and marine animal products (Table 2) and antimicrobial/antitumor higher plant and terrestrial animal products (Table 3 ).

In the followings the specific features and characteristics of microbial compounds will be discussed exclusively.

It is interesting that the overlapping between the products of the main groups of producers is negligible. There are only a relatively few cases when simple, low molecular compounds, are produced by microbes and higher plants, alike (simple aromatics, heterocycles, clavine alkaloids, some flavonoids, etc.), however, the symbiotic origin cannot be excluded. The overlapping of relatively large, complicated microbial and higher plant/animal products is practically negligible. Important to note that the overlapping between products of the main groups of microbial producers (fungi and actinomycetes e.g.) is also very rare, less than $1 \%$. The overlapping between Streptomyces and the taxonomically similar rare actinomycetales products is more frequent, but it is still only about $10 \%$.

\section{The Research of Microbial Metabolites (Statistical Evaluations)}

Obviously various actinomycetales, first of all the Streptomyces species and filamentous fungi, and to a lesser extent several bacterial species are the most noteworthy producers both in respect of numbers, versatility and diversity of structures of the produced metabolites.

The significance and frequency of these main types of microbes as producers of bioactive metabolites had varied significantly during the last decades. In the beginning of the antibiotic era the fungal (penicillin, griseofulvin) and bacterial (gramicidin) species were in the foreground of the interest, but after the discovery of streptomycin and later chloramphenicol, tetracyclines and macrolides the attention turned to the Streptomyces species. In the fifties and sixties the majority $(\sim 70 \%)$ of antibiotics were discovered from these species. In the next two decades the significance of the non-Streptomyces actinomycetales species (rare actinos) were increased, up to a $25 \sim 30 \%$ share of all antibiotics. (Fig. 1) From the early nineties the number of bioactive compounds isolated from various filamentous and other microscopic and higher fungal species had continuously increased up to more than $50 \%$ by the turn of the millenium (2000). The interest to bacteria in the recent years had only slightly increased. Simultaneously, the ratio of actinomycetal compounds naturally had definitely decreased. (see in Fig. 1a)

The most characteristic and a little bit surprising feature of the recent years just is this declining representation of the formerly exhaustively investigated actinomycetes. Their share among all microbial products presently is only $30 \sim 35 \%$, in contrast to the $75 \sim 80 \%$ share from the sixties to eighties. The shift in their apparent participation, is rather the result of the favoured fashion of the fungal screening in some laboratories. Presently the claim for new microbial pharmacophores led to the shift of the efforts in most places towards the discovery of fungal products from the large pool of untapped fungal world. I think, however, the present slight over-estimation of the capability of fungi is rather a periodic phenomenon. It is very likely that the changes in the interest towards the favorite microbes (as happened with the actinomycetes in the earlier years) depends on new expectations, the changing needs in the human therapy, and probably sometimes on fashion. It is also likely that for numerous reasons the actinomycetales, 
Table 4 Approximate number of bioactive microbial metabolites according to their producers and bioactivities

\begin{tabular}{|c|c|c|c|c|c|}
\hline \multirow{3}{*}{ Source } & \multicolumn{5}{|c|}{ Bioactive secondary microbial metabolites } \\
\hline & \multicolumn{2}{|c|}{ Antibiotics } & \multicolumn{2}{|c|}{ Bioactive metabolites } & \multirow{2}{*}{$\begin{array}{c}\text { Total bioactive } \\
\text { metabolites }\end{array}$} \\
\hline & Total & (with other activity) & no antibiotic activity & $\begin{array}{l}\text { (antibiotics plus } \\
\text { "other bioactives") }\end{array}$ & \\
\hline Bacteria & 2900 & $(780)$ & 900 & (1680) & 3800 \\
\hline Eubacteriales & 2170 & $(570)$ & 580 & $(1150)$ & 2750 \\
\hline Bacillus sp. & 795 & (235) & 65 & $(300)$ & 860 \\
\hline Pseudomonas sp. & 610 & (185) & 185 & $(370)$ & 795 \\
\hline Myxobacter & 400 & $(130)$ & 10 & $(140)$ & 410 \\
\hline Cyanobacter & 300 & (80) & 340 & $(420)$ & 640 \\
\hline Actinomycetales & 8700 & $(2400)$ & 1400 & $(3800)$ & 10100 \\
\hline Streptomyces sp. & 6550 & (1920) & 1080 & $(3000)$ & 7630 \\
\hline Rare actinos & 2250 & $(580)$ & 220 & $(800)$ & 2470 \\
\hline Fungi & 4900 & $(2300)$ & 3700 & $(6000)$ & 8600 \\
\hline Microscopic fungi & 3770 & (2070) & 2680 & $(4750)$ & 6450 \\
\hline Penicillium/Aspergillus & 1000 & $(450)$ & 950 & $(1400)$ & 1950 \\
\hline Basidiomycetes & 1050 & $(200)$ & 950 & $(1150)$ & 2000 \\
\hline Yeasts & 105 & (35) & 35 & (70) & 140 \\
\hline Slime moulds & 30 & (5) & 20 & (25) & 60 \\
\hline Total Microbial & 16500 & $(5500)$ & 6000 & $(11500)$ & 22500 \\
\hline Protozoa & 35 & (10) & 5 & (45) & 50 \\
\hline
\end{tabular}

besides the fungi, will remain equally important and promising producers in the future.

The serious problems of chemotherapy, the again and again increasing resistance of bacteria and fungi, the newly emerging old and new pathogens (mycobacteria, anaerobs, etc.), the high mortality of some common bacterial diseases, the problems of viral infections and neoplastic diseases etc., all requires new agents which, I strongly believe, greatly will be based on sophisticated study of the until now less known new, rare actinomycetales as producers. I think, the fungal products will gain eminent importance mostly in the pharmacology and perhaps agriculture.

In Table 4 the numbers of the all antibiotics (without other activities), the numbers of the antibiotics exhibiting additional "other" bioactivities (in parenthesis), and the "other bioactive" metabolites as well as the total numbers are summarized according to the main producer types and several specific producer species.

In the group of unicellular bacteria the most frequent producers are the Bacillus ( $\sim 800$ compounds are produced by these species), and Pseudomonas species (near to 800 compounds), followed by the entero- and lactobacillii, and streptococcii, each representing over hundreds of produced compounds. They commonly produce peptides or modified peptides, simple heterocycles (phenazines) and aliphatic compounds (fatty acid derivatives), but the occurrence of other, complicated chemical types (e.g. macrolactones) is relatively rare. The recently emerging Cyanobacter and Myxobacter species, producing 640 and 410 bioactive compounds, respectively produce chemically more diverse metabolites and several interesting compounds, such as the promising antitumor agent epothilone.

Notwithstanding the recent drop, the predominant part, $45 \%$ of the presently known bioactive microbial metabolites, over 10000 compounds were still isolated from various actinomycetales species, $34 \%$ from Streptomyces and $11 \%$ from the rare actinos. The most frequent producers, the Streptomyces species produces 7600 compounds ( $74 \%$ of all actinomycetales), while the rare actinomycetes represent $26 \%$, altogether 2500 compounds. The representation of rare actino products in 1970 was only 5\%. In this group Micromonospora, Actinomadura, Streptoverticillium, Actinoplanes, Nocardia, Saccharopolyspora and Streptosporangium species are the most frequent producers, each produces several hundreds of 
Table 5 Number of actinomycetales species producing bioactive microbial metabolites

\begin{tabular}{|c|c|c|c|}
\hline Streptomycetaceae: & & Thermomonosporaceae: & \\
\hline Streptomyces & $\sim 8000$ & Actinomadura & 345 \\
\hline Streptoverticillium & 258 & Saccharothrix & 68 \\
\hline Kitasatosporia & 37 & Microbispora & 54 \\
\hline Chainia & 30 & Actinosynnema & 51 \\
\hline Microellobosporia & 11 & Nocardiopsis & 41 \\
\hline \multirow[t]{2}{*}{ Nocardioides } & 9 & Microtetraspora/Nonomuria & $26 / 21$ \\
\hline & & Thermomonospora & 19 \\
\hline Micromonosporaceae: & & Micropolyspora/Faenia & $13 / 3$ \\
\hline (Actinoplanetes) & & Thermoactinomyces & 14 \\
\hline Micromonospora & 740 & Thermopolyspora & 1 \\
\hline Actinoplanes & 248 & Thermoactinopolyspora & 1 \\
\hline Dactylosporangium & 58 & & \\
\hline Ampullariella & 9 & Mycobacteriaceae: & \\
\hline Glycomyces & 2 & (Actinobacteria) & \\
\hline Catenuloplanes & 3 & Nocardia & (357) \\
\hline \multirow[t]{2}{*}{ Catellatospora } & 1 & Mycobacterium & 57 \\
\hline & & Arthrobacter & 25 \\
\hline Pseudonocardiaceae: & & Brevibacterium & 17 \\
\hline Saccharopolyspora & 131 & Proactinomyces & 14 \\
\hline Amycalotopsis/Nocardia & $120 / 357$ & Rhodococcus & 13 \\
\hline Kibdellosporangium & 34 & & \\
\hline Pseudonocardia & 27 & Other (unclassified) species: & \\
\hline Amycolata & 12 & Actinosporangium & 30 \\
\hline Saccharomonospora & 2 & Microellobosporia & 11 \\
\hline \multirow[t]{2}{*}{ Actinopolyspora } & 1 & Frankia & 7 \\
\hline & & Westerdykella & 6 \\
\hline Streptosporangiaceae: & & Kitasatoa & 5 \\
\hline (Maduromycetes) & & Synnenomyces & 4 \\
\hline Streptosporangium & 79 & Sebekia & 3 \\
\hline Streptoalloteichus & 48 & Elaktomyces & 3 \\
\hline Spirillospora & 11 & Excelsospora & 3 \\
\hline Planobispora & 10 & Waksmania & 3 \\
\hline Kutzneria & 4 & Alkalomyces & 1 \\
\hline \multirow[t]{5}{*}{ Planomonospora } & 2 & Catellatospora & 1 \\
\hline & & Erythrosporangium & 1 \\
\hline & & Streptoplanospora & 1 \\
\hline & & Microechinospora & 1 \\
\hline & & Salinospora & 1 \\
\hline
\end{tabular}

antibiotics. In Table 5 the numbers of actinomycetales species, including the all rare actinos, known to produce bioactive metabolites, are summarized.

These fastidious organisms, the rare actinos, produce perhaps the most diverse and most unique, unprecedented, sometimes very complicated compounds exhibiting excellent antibacterial potency and usually low toxicity. It is interesting that several chemical types, such as simple terpenoids or benzenoids are almost completely absent from this compounds. In this group of metabolites there are numerous practically very important compounds such as gentamicins, erythromycins, vancomycin, or rifamycin. Numerous recently introduced chemotherapeutic and agricultural agents (ziracin, dalbavacin, spynosin), are also rare actino products. It is noteworthy that the vancomycinristocetin type complicated glycopeptides are produced almost exclusively by various rare actino species.

Presently more than 50 rare actinos are known as producers of the 2500 bioactive compounds, but in 1970 only 11 rare actino species, producing altogether 50 compounds, were known. The number of all taxonomically described rare actinos today is close to 100 but this number, 
due to the recently developed genetic and isolation techniques will, be in all means quickly increasing.

The present relatively low occurrence of rare actinos, in contrast to Streptomyces species is derived from the facts that they are hard to isolate from the environment and difficult to cultivate and maintain under conventional conditions. These are reasons why these species are still regarded to be rare. Recently however advanced isolation techniques have been developed, and with these techniques from an environmental sample the overwhelming majority of these rare species could be isolated $[15,16]$.

In the light of our accumulated knowledge and in the statistical data, however, the potency of the Streptomyces species should not be underestimated. Their capacity to produce promising new compounds will certainly be unsurpassed and for a long time and they still have been producing the majority of the chemotherapeutically applied antibiotics.

Among fungal species, the various microscopic (filamentous) fungi (ascomycetes, fungi imperfecti, etc.) are the most frequent producers with about 6400 produced compounds. From the most common ascomycetes, namely from Aspergillus, Penicillium and Fusarium species 950, 900 and 350 compounds have been isolated, respectively. Besides them several other filamentous and endophytic species (Trichoderma, Phoma, Alternaria, Acremonium and Stachybotrys), are also good producers, each produces several hundreds of bioactive compounds. From higher fungal species-basidiomycetes or mushroomsexemplified by Ganoderma, Lactarius or Aureobasidium species, altogether about 2000 active compounds have been derived. From yeasts, only 140 and from Myxomycetes (slime moulds) species 60 bioactive metabolites have been isolated.

The chemically relatively simple fungal compounds, over the antibiotic activities frequently exhibit diverse biological effects, mainly phytotoxic and pharmacological activities. We should not forget, the great practical and historical importance of beta-lactams (penicillins, cephalosporins), the cyclosporin, and various statins (mevinolin, compactin, lovastatin, pravastatin, atrovastatin), which are all fungus derived compounds.

Recently it is unquestionable that the interest to all types of fungal species, but mainly to endophytic and the so called marine fungi as possible sources of new bioactive compounds is highly increasing. The expansion of the very quick new screening methods led to the appearance of the increasing number of "unidentified" fungus as bioactive metabolite producers (over 250 new metabolites in the last two years), especially in the patent literature. It indicates the high speed of isolation, and identification/patenting process of new fungal products, and the long time need for taxonomical identification of new fungal species.

\section{Biological Activity (Biodiversity)}

\section{Functions of Secondary Metabolites (Biological Functionality)}

It seems that presently only the biological activity (antimicrobial, antitumor, antiviral, pharmacological, and similar activities) is the guiding line which connects the bioactive microbial metabolites, distinguishing them from the other "inactive" natural products, which evidently emerges an essential question. Is there any general difference between these "bioactive" compounds and the other "inactive" natural products or secondary metabolites in any other point of view? Is perhaps the biological activity some kind of differentiating property? Their origin, the types of their producer species or their biosynthetic pathways, may be different? Are their production methods or isolation routes perhaps unique? Do their physical or chemical properties, perhaps chemical structures show significant differences?

The answer is no, definitely not!

It is true, that several uncommon, specific chemical structures, structural elements, and unique chemical groups, (macrolactone, cyclopeptide skeleton, unusual functional groups, etc.), are more frequently occurring among bioactive, than the "inactive" metabolites, but these structural features are also occurring in the group of microbial compounds considered presently as inactives. It seems, these unique structural features are rather belonging to the whole group of secondary microbial metabolites than specifically to the distinct groups of bioactive products. The well known, widespread but specific chemical structures may be connected with the antibiotic activity and other, perhaps until now less characteristic or unrecognized chemical structural elements may be connected with other, new types of activities.

It is my strong belief that there is no any reason to exclude the possibility of the existence of some sort of biological activity (interaction with some other living organisms) of any chemical compound derived by secondary metabolism of microorganisms (or perhaps macroorganisms), which are known today or may be discovered in the future. In other words, I mean, that all of the described and possible microbial secondary metabolites have some kinds of inherent activity but in many cases these activities have not yet been discovered; simply we do not know what they are. Only the methods to detect their 
Table 6 Microbial interactions

\begin{tabular}{ll}
\hline Microbe-Microbe & Antimicrobial antibiotics, microbial regulators, growth factors, signaling compounds, mating \\
hormones, etc. & Insecticides, miticides, antiparasitic compounds, algicides, antifeedants, (invertebrates) repellents, \\
$\begin{array}{l}\text { Microbe-Lower Animals } \\
\text { (invertebrates) }\end{array}$ & molluscicides, anti-worm agents, etc. \\
Microbe-Higher Plants & Herbicides, phytotoxins, plant growth regulators, chlorosis inducers, phytoalexins, etc. \\
$\begin{array}{c}\text { Microbe-Mammalians } \\
\text { (humans) }\end{array}$ & Antitumor antibiotics, pharmacologically active agents, enzyme inhibitors, (humans) immunoactive, \\
CNS-active, etc. agents, feed additives, etc.
\end{tabular}

possible, perhaps until now unknown type of activity, has to be developed.

There is no reason to suppose that the majority (if not all) of the natural products including microbial metabolites should not exhibit some kind of biological function. Do not forget that hundreds of presently known bioactive metabolites originally was discovered as "inactive", natural product and their activity was only discovered later, investigating them with new more specific methods, or reisolated them (sometimes from different species) using more sensitive methods, based on absolutely new principles. In our days, in fact, there would not be any reason to talk about "bioactive" or "inactive" secondary metabolites and treat them separately. They represent only temporary categories.

The secondary metabolites may be evolved in nature as some kind of response to the effects of the environment, including living and physical environments. Nature being a sophisticated, versatile and energetic combinatorial chemist all over the times yields by infinite number of different and unpredictable ways, a series of exotic and effective structures, which have ever been made in laboratories. The natural product biosynthesis, driven enzymatically from a huge gene pool has evolved over billions of years. As a result of this evolutionary process the secondary metabolites certainly exists in coded form in the nucleic acid world as some kinds of regulators or effectors. I hope, that with the benefit of widening our knowledge about biosynthetic genes, in the future we will be able to clarify the role and functions of these latent activities and exactly prove my hypothesis, namely the necessity of the universal existance of the inherent biological activity of secondary metabolites. The correct answer to these questions may explain the unknown reason of the existence of secondary metabolites, the purpose of their production and our still missing exact knowledge of their real role in the life of their producers.

Taking into consideration some other ecological points of view, without claiming the precise explanation of the reason of existence of secondary metabolites, it may be suppose that the microbial secondary metabolites represents a kind of chemical interface-manifested in diverse interactions - between microbes and the rest of the world. The scope of these interactions certainly is tremendous. Antagonistic, synergistic, regulatory or modulatory and any other biochemical or either biophysical interactions represent the connections between microbesthrough their secondary metabolites-and other living systems and with the physical environment. Interactions of microbes with other microbes and non-microbial systems: higher plants, lower animals or mammalian systems, including humans are the playing ground of the secondary metabolites. These interactions may be summarized as illustrated in Table 6 covering the whole area of known biological activities of microbial metabolites, at the same time representing their possible practical applications. The wide interpretation of these interactions helps to understand the reason why the microbes-by means of their chemical products-exhibits so wide range of bioactivities.

A very important and not negligible consequence of this view is, that the biochemical targets (the places of these interactions) found in eukariotic microbes, plants, invertebrates and mammals, are equally part of the eukariotic biochemistry, and therefore they are highly overlapping. This fact creates excellent challenges to expand the possibility to discover compounds, on the basis of one target, which may be applied in various areas, for example in the medicine or agriculture, alike.

\section{Bioactivities of Secondary Metabolites}

The presently known secondary microbial metabolites, exhibit a great numbers of diverse and versatile biological effects, first of all antimicrobial activities. In the scientific literature already hundreds of different pathogenic and other microbes (Gram-positive, Gram-negative bacteria, fungi, yeasts, etc.) are described as test organisms in the direct activity-based screenings. The most frequent test organisms were Bacillus subtilis, Staphylococcus aureus, Micrococcus (Sarcina) lutea, Escherichia coli, 
Table 7 Bioactivity types of microbial metabolites numbers of discovered bioactivities

\begin{tabular}{|c|c|}
\hline Type of activity & $\begin{array}{l}\text { Numbers of discovered } \\
\text { bioactivities }\end{array}$ \\
\hline \multicolumn{2}{|l|}{ ANTIBIOTIC ACTIVITIES: $\quad(16500$ compounds) } \\
\hline \multicolumn{2}{|l|}{ Antimicrobial Activity: } \\
\hline Antibacterial: Gram-positive & $11000 \sim 12000$ \\
\hline Gram-negative & $5000 \sim 5500$ \\
\hline Mycobacteria & $800 \sim 1000$ \\
\hline Antifungal: & $3000 \sim 3500$ \\
\hline Phytopathogenic fungi & $1600 \sim 1800$ \\
\hline Other fungi & $3800 \sim 4000$ \\
\hline Antiprotozoal: & $\sim 1000$ \\
\hline \multicolumn{2}{|l|}{ Chemotherapeutic activity: } \\
\hline Antitumor (cytotoxic) & $5000 \sim 5500$ \\
\hline Antiviral & $1500 \sim 1600$ \\
\hline \multicolumn{2}{|l|}{ OTHER BIOACTIVITIES: (11500 compounds) } \\
\hline \multicolumn{2}{|l|}{ Pharmacological Activity: } \\
\hline Enzyme inhibitor & $3000 \sim 3200$ \\
\hline $\begin{array}{l}\text { Immunological activity } \\
\text { (suppressive, modulatory) }\end{array}$ & $\sim 800$ \\
\hline $\begin{array}{l}\text { Biochemical activity } \\
\text { (DNS, tubulin, mitotic, etc.) }\end{array}$ & $\sim 1000$ \\
\hline $\begin{array}{l}\text { Other (antagonistic, modulatory, } \\
\text { antiinflammatory, etc.) activities }\end{array}$ & $2000 \sim 2500$ \\
\hline \multicolumn{2}{|l|}{ Agricultural Activity: } \\
\hline $\begin{array}{l}\text { Pesticide (antiparasitic, algicide, } \\
\text { amoebicide, etc.) }\end{array}$ & $900 \sim 1000$ \\
\hline $\begin{array}{l}\text { Herbicide (phytotoxic, plant growth } \\
\text { regulatory, etc.) }\end{array}$ & $1800 \sim 1900$ \\
\hline Insecticide/Miticide/Larvicide/Deterrent & $1100 \sim 1200$ \\
\hline Feed additive, preservative & $300 \sim 400$ \\
\hline Other Activities & $\sim 1000$ \\
\hline $\begin{array}{l}\text { Microbial regulators (growth factors, } \\
\text { microbial hormones, morphogens) }\end{array}$ & $\sim 500$ \\
\hline Biophysical effects (surfactants, etc.) & $\sim 300$ \\
\hline
\end{tabular}

Pseudomonas aeruginosa, Saccharomyces cerevisiae, Candida albicans and others. Antiviral tests, inhibition of viral enzymes, activities connecting with neoplastic diseases from simple cytotoxicity assay methods (P-388, $\mathrm{KB}, \mathrm{L}-1210$ cell lines) to angiogenesis inhibition, etc., are used most frequently for detection of other, nonantimicrobial activities of metabolites.

However, the infectious diseases are mainly treated with natural antibiotics and their derivatives, while still the majority of drugs applied in the so called physiological diseases are synthetic products. The challenge for natural medical products to treat these diseases is huge. There is an urgent need in this area to identify totally new active chemotypes at least as lead compounds for effective drug development.

The list of non-antibiotic biological activities used in the new screening projects presently covers more than one thousand different types of bioactivities; cell based-, receptor binding- or enzymatic assay methods and many other specific tests and targets. In our days more and more newer, sophisticated and diverse assays are used worldwide in various screening protocols.

The main types of bioactivities, the frequency of their occurrence in the scientific literature, according to historical and practical point of views, may be summarized in the Table 7.

We have to note that in this Table, and in most cases the statistical numbers are indicating the discovered and published bioactivities or other characteristics, and not the really existing ones, therefore some statistical data (percentage of bioactivities, discussed in the next section) may be somewhat misinforming due to the frequent exclusive use of the specific tests, assays and models in various screening projects. Missing data in the publications, e.g. do not exclude the possible existence of any activity or characteristics.

In practical point of view most of the presently known non-antibiotic bioactivities may be classified as:

- pharmacological-biochemical or medical activity,

- agricultural activity,

- regulatory, biophysical and other activities

The first type of "other" bioactive compounds with possible medical activity in the largest number covers the potentially very important and promising metabolites with enzyme inhibitory activities. Presently over 3000 compounds known to possess inhibitory activity against about 300 to 350 various enzyme systems. The agriculturally active compounds, of course may also include enzyme inhibitory compounds. There are about 800 immunoactive compounds (immunosuppressive, immunostimulatory), hundreds of compounds with the most diverse regulatory; inhibitory, agonist and antagonist activity, including antiinflammatory/antioxidative, hypocholesterolemic, antimetabolite and various toxic (mycotoxic, etc.) action. The detected biochemical activities as tubulin (microtubule) assembly inhibitory, interferon inducing, antimitotic/antimitogenic, DNA damaging, antimutagenic effects, apoptosis inducing activity, angiogenesis inhibition, etc., are also frequently occurring. The assays detecting these activities show extremely large variations, and the range of the final physiological, biological, biochemical, phytochemical and microbiological effects include close to one thousand bioactivity categories. The compounds, discovered by these specific methods may be called 
(distinguishing them from the antibiotics) as microbial medicinal products or as "biopharmaceutins". I think to mention cyclosporin, lovastatin, or tacrolimus, is enough to point to the practical importance of these compounds.

Feeding billions of people, besides the health care, is one of the most urgent needs for humankind. The increasing of the agricultural production (including the animal husbandry), when at the same time environmental and economic requirements have to be taken into consideration, meaning the largest advantage to the microbial compounds over the synthetic chemicals. Most of the environmentally acceptable agricultural compounds are natural products or are derived by modification of natural product leads. The so called agricultural activities are including mainly pesticides (insecticide, miticide, larvicide, repellents, deterrents, antifeedants, antiparasitic, anthelminthic agents, cestocides, acaricides, nematocides, antiworm compounds, algicides, and other biocontrol agents), and herbicides (phytoactive agents: phytotoxins, plant growth regulators, germination inhibitors, allelochemicals, phytohormones, chlorosis inducers, etc.). Feed additives, preservatives, permittants/growth promoters represent the compounds used in the husbandry and veterinary. The market of environmentally acceptable herbicides, pesticides and fungicides reaches 50 billion $\$$ year, perhaps larger than the medical market. Avermectin, monensin and bialaphos are the most useful microbial compounds in this group.

Practically less important, but in respect of biological sciences, invaluable group of microbial regulatory compounds produced also in a great number by microorganisms. Specific metabolic inhibitors, growth factors, morphogenic and sporogenic agents, signal compounds, mycelium formation inducers, metabolite production stimulators, autoregulators, bacterial pheromones, mating inhibitors, differentiation inducers, siderochromes, etc. are included into this group. These compounds play a significant role in the life cycle of microbes and may be used in the bioassays or as biochemical reagents. Compounds having biophysical effects are biosurfactants, biofloculants, chelate and ionchannel forming agents, mineral scavengers, various ionophores, photoactive compounds, radioprotectants, etc.

\section{Main Types of Activities (Statistical Evaluations)}

\section{Antibiotic activities}

Some $60 \%$ of the presently known bioactive microbial metabolites, about 14000 compounds, exhibit antimicrobial (antibacterial, antifungal, antiprotozoal), approximately 5000 compounds have antitumor and about 1500 compounds exhibits antiviral activity. Of course, these observed activities may be significantly overlapping.

Additionally to antibiotic activities, some 11500 microbial metabolites, half of the all compounds, possess some kind of additional (or exclusive) "other"-nonantibiotic-bioactivities. Among them there are about 6000 metabolites without any recognized antimicrobial activity exhibiting exclusively some kinds of "other" biological activities. They are the so called "other bioactive" metabolites. The numbers of antibiotics ("classical" antibiotics) and the "other bioactive" metabolites according to their origin, were summarized in the Tables 2 and 4 . As derived from the numbers including in these Tables, in total 16500 compounds, $73 \%$ of all bioactive metabolites may be considered strictly as antibiotics.

In the group of antibiotics the inhibitory activity against Gram-positive, Gram-negative and mycobacteria exist in $66 \%, 30 \%$ and $5 \%$; $(10900, \sim 5000$, and 350$)$ of the compounds, respectively. Altogether 5600 compounds (34\%) shows antifungal activity; for which $21 \%$ (3500) are active against yeasts, $11 \%$ (1800) against phytopathogenic fungi and 24\% (4000) are active against other fungal species. About 2000 compounds, e.g. the polyene antibiotics, exhibit exclusively antifungal/antiyeast activity.

The combination of various antimicrobial and other activities shows a wide variation. A great part of antibiotic compounds exhibit exclusive activity against Gram-positive bacteria ( $\sim 30 \%$ of all), but there are "broad antibacterial spectrum" compounds with activity against Gram-positive, Gram-negative and mycobacteria (15\%) and there are the broadest spectrum compounds with additional antifungal activity (12\%). Only $1.5 \%$ of the compounds (some 250 metabolites e.g. the polymyxins), exhibits activity only against Gram-negative bacteria. The number of antimicrobial compounds with additional antitumor and/or antiviral activities is about 3000 . There are about 500 compounds showing exclusively antitumor activity and approximately $100-100$ compounds are active solely against viruses or protozoa.

Several significant differences occur in the frequency of various antibiotic activities according to their microbial origin. The actinomycetales and bacterial products exhibit primarily antimicrobial activities. About $74 \%$ of all actinomycetales products-over $80 \%$ of the rare actino products-and similarly $70 \sim 75 \%$ of various bacterial products exhibit antibacterial and/or antifungal activities. In contrast, only $40 \sim 45 \%$ of the all fungal products have some kinds of antimicrobial, frequently antifungal, activities. The antitumor activity shows less significant differences in its distribution; namely 30, 24 and $27 \%$ for actinomycetes, bacterial and fungal products respectively, have antitumor activity. Only the $40 \%$ rate of the antitumor 
Myxobacter products is remarkable.

Other bioactivities:

As it has been seen before in total 11500 microbial metabolites possess "other" non-antibiotic activity including approximately 6000 compounds which exhibit exclusively these activities, without additional (but perhaps undiscovered) antibiotic activities (Table 4). It is significant that in this group about 3700 fungal, only 1400 actinomycetales, about 900 bacterial, but 340 ! cyanobacterial products occur.

As it can be seen from these numbers the frequency of occurrence of the "other" biological activities is highly variable. The $38 \%$ of actinomyces products, but surprisingly only $32 \%$ of the rare actino products, and $34 \sim 36 \%$ of various bacterial products possess some kinds of other-pharmacological or agricultural-activities. In contrast this ratio is very high, as high as $72 \%$, in the products of microscopic fungi and $58 \%$ of the compounds isolated from basidiomycetes. This high percentage of microscopic fungal products derived from the frequently occurring pharmacological and phytotoxic-phytoregulatory ( $\sim 1500$ fungal compounds exhibits some kinds of "phytoactivity") activities. Interestingly only $2.6 \%$ ! (263) of the all actinomycetales metabolites show phytotoxic activity. Also noteworthy that the proportion of other activities (mainly enzyme inhibitory activities) in the case of Cyanobacter metabolites is relatively high; $67 \%$, close to those of the fungal products, while among metabolites of Bacillus species is very low, only $7 \%$.

These significant differences are certainly derived from the taxonomic position, the different biosynthetic capability of the producers, therefore from the chemical types of compounds produced by these species. It has to be taken into account, however, that the relatively low statistical occurrence ( 38 and $32 \%$ ) of the other bioactivities among the actinomycetes, and perhaps bacterial products (34 36\%) may be derived from the existence of a great number of old known compounds, those were almost exclusively screened and discovered only on the basis of their antimicrobial activity. In the last decade over $60 \%$ of the all discovered new actinomycetales derived compounds exhibit additional, "other", mainly enzyme inhibiting and other pharmacological activities, points to the similar profitability of these microbes.

\section{Chemical Structures (Chemical Diversity)}

\section{Increasing the Biosynthetic Diversity}

In the area of natural products there is no any other group where so wide range of specific and complex chemical structures, with fascinating array of diverse, unique functional groups occur, than in the group of antibiotics and other bioactive microbial secondary metabolites. Compounds in this group cover all types of organic compounds from the simple acrylamidine (MW: 72) to the most complicated structures, such as the macrocyclic colubricidin (MW: 2154), which includes large glycosilated macrolactone ring, pyridine and pyrrole moieties. The polycyclic tetropetalone-A [26] contains one of the most complicated heterocyclic ring system with quinone and glycosidic functionalities or the macrocyclic versipelostatin [27] also have fascinating new structures.

The majority of the unique collection of molecular skeletons of microbial secondary metabolites, never occur in any chemical libraries. As it is estimated, this part is over $40 \%$ of the natural product diversity which means that at least half of the bioactive microbial metabolites had never been synthesized [31].

Unfortunately, however, notwithstanding the ten thousands of known fantastic structures, it looks like that in our days serious difficulties are emerging from the insufficient chemical variability of the presently existing compounds used in the human therapy. The escalation of clinical resistance, the acquired multidrug resistance (MDR), the emerging new pathogens, series of neoplastic and viral diseases, HIV, Ebola, etc., all represents an ever increasing and returning problems in the chemotherapy. The challenges of the existing almost 25000 to 30000 clinically described human diseases, mainly are "physiological" diseases, highly require the further increasing of this chemical diversity [14]. Presently we can treat symptomatically only a smaller part, and can cure effectively only a minor portion of these diseases. The most important challenge of the future is how we can discover more and more chemically and hopefully mechanistically new agents to satisfy these needs of human therapy.

The real problem is: how we can access most effectively to the natural chemical diversity. It is very likely that the effectivity and fruitfulness of the classical antibiotic research by the end of the millenium are definitely exhausted. In the last ten years, in spite of the discovery of the great numbers of new compounds, less totally new type of structures were discovered than in the previous decades. The overwhelming majority of new compounds, are 
composed of new variations and arrangement of the old known structural elements. It is true that the structural variations, the unique combinations of rare moieties and skeletons of natural products due to the extremely versatile biosynthetic capacity (extensive branching, series of alternative reactions, isomerisation, condensations, polymerization, oxidation, alkylation, etc.) of the microbes, first of all actinomycetes, are inexhaustible. This specific nature of the microbial biosynthesis, led to the isolation of a great number of analogs and a series of homologues, the so called minor components. A great part of "new" compounds discovered in the last decades (about 30\%) were this kind of minor compounds. For example already in 1977 from a gentamicin producing Micromonospora species we were able to isolate 50 minor components [28]. This phenomenon playing an important role in the exponential increasing of the number of described metabolites, but unfortunately does not mean significant practical advantages.

The reason of the declining effectivity to obtain new chemical types, in general may be the result of the

- exhaustion of the biological sources or rather the

- imperfect screening methodologies.

Certainly the second reason is the critical one. Nature as a source of new chemicals is supposed to be almost inexhaustible (see later), but the classical screening methods used for long time does not meet the recent requirements of human therapy and agriculture. All chemotherapeutic agents used today belong to a limited number of chemical types (or close derivatives) discovered in the past by the classical methods. Still in our days for time to time several interesting compounds with new structural types, (nomofungin, TMC-95, colubricidin, vancoresmycin, treponemycin) are being discovered, but most of the new discoveries are variations on the theme. The recent introduction to the chemotherapeutic praxis several "old", narrow spectrum compounds, with known structural types, (pristinamycin, ziracin, daptomycin, echinocandin, ramoplanin, sordarin, etc.) represents some progress. One of the most interesting, new "discovery" also goes back to several decades. The original isolation of the antibiotic LL-AC98 happened in 1970, but its original new structure and possible practical usefulness has been discovered only recently. This compound, renamed as mannopeptimycin [29] is an interesting cyclopeptide containing mannose side chains and imidazole moiety. This exciting molecule has a relatively new type of structure and promising antimicrobial properties with significant activity against numerous resistant bacteria [30]. Another example is the recent introduction of the hundred-year old mycophenolic acid derivative (Mofetil) as effective immunosuppressant. This and several other examples (genetic modification of glycopeptides and macrolides e.g.), illustrate the significance of the reinvestigation of old compound "libraries", and the possibilities of the combinatorial biosynthesis, but these approaches do not mean drastic break-through. The total renovation of the screening projects, which was, in fact, an urgent necessity already several years ago, is unavoidable.

The first steps of this new revolution in the last several years fortunately have been already gradually accomplished. The main task of the first years of this century is no doubt the development of fundamental principles of new, adequate strategies for the new drug discoveries on the basis of absolutely new principles.

Competitiveness between natural products and syntheticcombinatorial libraries

New compounds may be prepared by chemical synthesis or may be isolated from the nature. The newly emerged combinatorial chemistry, besides the capacity to produce new compounds itself, in all means has less chances to produce useful leads for new drugs. I strongly believe, that natural resources will provide structurally and mechanistically new molecules serving as useful direct drugs or lead compounds, with better chances, than any chemical approaches. The pharmacological industry until now has synthesized several millions (according to some estimations about 3 to 4 millions) new organic chemical structures, but only a negligible part of them (no more than one from several ten-thousands, $\sim 0.001 \%$ ) became accepted drug. From the ten thousands of known microbial metabolites about $150 \sim 160(\sim 0.2 \sim 0.3 \%)$ compounds became practically utilized therapeutic product and about the same number proved to be successful lead compound, indicating the more than two order of magnitude higher effectivity of this approach.

If we are comparing the whole collection of known natural products, as recently widespread called as the library of natural products, including the bioactive microbial metabolites, with the random libraries of synthetic and combinatorial chemistry, it is impossible not to learn that the screening of microbial products libraries is more efficient as regards to the discovery of new, active compounds especially those that are currently beyond the economic capability of chemical synthesis. The inherent molecular diversity of natural products far outweighs to that of any chemical-combinatorial libraries ${ }^{31)}$. Perhaps only the combination of the natural product libraries with these chemical libraries, may provide more promising chances for the additional target based screenings, that is say to discover new, perhaps more active, useful derivatives 
or analogues of the natural products.

Natural products usually result new mechanisms of action and exhibit novel therapeutic activities with higher probability than the synthetic or combinatorial compounds. The reason of the higher efficiency of natural products research is perhaps the fact that the natural products, in contrast to these random collections of chemicals, are somewhat prescreened by nature and they are already evolved for specific biological interactions. Furthermore, in contrast to the synthetic compounds, the products of microbes have many similarities with metabolites of other living systems, e.g. mammalian systems, giving higher chances to exhibit drug-like action capable of being absorbed and metabolized, therefore requiring minimal modification to develop an effective, orally active and easily marketable product.

It was thought by somebody several years ago that combinatorial chemistry will replace the natural product research. The irrationality of this nonsense idea, I think, by our days is evident. Similarly to the overestimated capability of the Rational Drug Design (RDD) or the Structure Activity Relationship (SAR) approaches it has to be emphasized that the combinatorial chemistry has only complementary and not replacing role. It may assist to develop favorable derivatives of lead compounds originally derived by screening from microbial or other natural sources. They help only to modify the structurally and mechanistically new, inherently active natural compounds in respect of the absorption, transport and uptake, make them in vivo more effective in the host.

It is unquestionable that the microorganisms represent the biggest chance to obtain further medically, veterinary and agriculturally useful compounds, which may serve not only as direct drugs or pesticides/herbicides but also as lead compounds for structural modifications and templates for the rational drug design. It is an important point of view that in respect of the whole natural product research the microbes, over the higher plant and marine animal sources, give better chances for the satisfactory scale up.

Personally speaking, the beauty of the architecture of the unprecedented structures of natural products, never emerged in the mind of any organic chemists, always amazed me. It is my other strong belief that in most respects nature is the first. Today the possibilities of organic chemistry, both in the fantasy of chemists and in general effectivity, are far behind nature. Chemists today still are able only to copy nature.

\section{Structural Features of Microbial Metabolites (Statistical Evaluations)}

The detailed discussion of the various structural types and the illustration of several new fascinating chemical structures discovered in the recent years exceeds the space limitations of the present review and some of these interesting structures are already illustrated in several specific reviews $[6,8,11,13,24]$. Instead, I would like to summarize some simple statistical data regarding the general chemical properties of microbial metabolites including antibiotics.

Altogether more than $90 \%$ of all known bioactive secondary metabolites, (more than 95\% those discovered after 1990) have known chemical structures. In the last decade due to the development of organic synthetic methods the total synthesis of more and more antibiotic are already published. Today almost $40 \%$ of known microbial metabolites (antibiotics) have been synthesized but most of these synthetic routes have only scientific value. Presently more publications describe the synthesis of known natural products, than the isolation of new compounds.

Antibiotics, and in general the bioactive microbial metabolites, have several common structural features. The most characteristic structural elements, unquestionably are the cyclopeptide/cyclopeptolide, depsipeptide frameworks and the various macrocyclic lactone/lactam ring systems, especially in the actinomycetales products. These types are rare in synthetic libraries and also infrequently occur in other, e.g. plant derived natural products.

The largest chemical group of antibiotics, are the amino acid derived diverse peptide type compounds, from the simple amino acid derivatives, including the $\beta$-lactams, to the high molecular proteides, covering about 5000 compounds. The next largest group is the diverse macrolactones, polylactones and ansalactones including simple, 8 to 12 membered lactones, macrolides, other macrocyclic lactones with 18 to 60 membered rings, and various condensed macrolactones, e.g. the milbemycins or cytochalasins, covering about 2400 to 2600 compounds. Various quinone derivatives with simple and condensed ring systems, covers some 1600 to 1800 compounds. Besides these main chemical types there are simple or complicated sugar derivatives, $\mathrm{N}$ - and $\mathrm{O}$-heterocycles, benzene and other aromatic derivatives, various alicyclic and aliphatic compounds including the terpenoids and various fatty acid derivatives.

Antibiotics covers the whole organic chemistry. In my chemical classification of the antibiotics [2,10], the compounds are systematized according to their chemical structures, enlisting about 1800 subcategories, classified 
the compounds according to a numerical nomenclature. Due to this pure chemical systematization, without the consideration of any biosynthetic point of view, the polyketides or e.g. acetate-mevalonate derived compounds are distributed to various chemical types.

The distribution of distinct chemical types of antibiotics according to their main producer types shows some remarkable features. All of the known (about 120) glycopeptide antibiotics are produced almost exclusively, and orthosomycins mainly, by the rare actinomyces species. The macrolides, polyene antibiotics, aminoglycosides, anthracyclines (each groups covering about 400 500 compounds), are all produced exclusively from actinomycetales species. Other smaller groups, such as the polyether antibiotics ( $\sim 250$ compounds); the novobiocin related glycosidic antibiotics, streptothricins, actinomycins and echinomycin-like quinoxalin peptides (each covers $80 \sim 120$ compounds); elfamycins, glutarimides, orthosomycins (each $50 \sim 60$ compounds), are also exclusively actinomyces products. The predominant part of the large, 18 to 60 membered macrocyclic lactone derivatives (including over 1000 compounds), ansa lactones $(\sim 150)$, benzanthraquinone derivatives $(\sim 200)$, thiostrepton-like thiazolyl peptides $(140)$, cyclopolylactones $(\sim 40)$, benzdiazepine antibiotics $(\sim 60)$, tetracyclines $(\sim 40)$, macrodi- and -tetrolides $(30 \sim 40$ comps.) are derived also from various actinomycetales species.

Peptaibols (300 400 compounds), grisan and scirpene derivatives, cytochalasins (all contains over hundred compounds) are produced exclusively by fungi. The betalactam antibiotics $(\sim 250)$, purine- $(\sim 130)$ and pyrimidine ( $\sim 240)$ glycosides, depsipeptides $(\sim 200)$ are derived from fungi and actinomycetales, and sometimes from bacteria, alike.

Microscopic fungi frequently produces small, 8 to 10 membered lactone ring compounds $(\sim 200)$, dilactones or condensed macrolactones,. macrodiolides, azaphilones, diketothiapiperazines, small terpenoids and simple benzene derivatives, alpha-pyrones, xanthone derivatives, simple benzo- or naphthoquinones, etc.

Several types of lipocyclopeptides (polymyxins), and the lanthibiotics (nisin type), are exclusively bacterial products. On the other hand, unicellular bacteria only exceptionally produce macrocyclic lactones (difficidin) and Cyanobacter and Myxobacter species do not produces macrolides, at all.

Noteworthy, that more than half $(56 \%)$ of all bacterial metabolites are some kinds of derivatives of amino acids; peptides, peptolides, polypeptides, etc.

The complicated large macrocyclic compounds are most frequently occurring in the group of rare actino $(21 \%)$ and
Streptomyces $(16 \%)$ products in contrast of the as low as $2 \sim 4 \%$ occurrence in the products of all other microbes. The occurrence of alicyclic, simple terpene derivatives in the fungal world is relatively high; $20 \sim 25 \%$ of all fungal products, while their share among actinomycetes and bacterial products is only $1 \sim 2 \%$.

The molecular weights of antibiotics is highly variable in the range from 72 to the several millions of macromolecular compounds. The most complicated and perhaps most versatile structures are derived from the actinomycetales species, first of all from the rare actinos, as reflected in the molecular weight distribution. The actinomycetales compounds usually medium to large size molecules with an average molecular weight of 400 to 800 (covering almost $60 \%$ of these compounds). In the 800 to 2000 molecular weight range the number of rare actino products is almost twice as that of Streptomyces products, namely 24 and 13 percents, respectively, indicating the higher representation of the more complicated structures. The fungal products have usually smaller molecular weight, they are less complex compounds with average molecular weight of 200 to 400 ( $56 \%$ of all). In the bacterial products the low molecular simple metabolites (MW: below 300) and the macromolecular peptides with molecular weight values over 1500 , are dominating.

It is somewhat surprising, and difficult to explain, that in the molecules of rare actino products the occurrence of halogen elements (chlorine and bromine) is more than three times higher than in those of Streptomyces products, namely $15 \%$ in contrast to the $4.5 \%$ of Streptomyces products. It is interesting also that in the products of seaweeds and marine animals, living in the chlorine rich sea water, the occurrence of bromine is significantly greater than the chlorine content, 25 and $15 \%$ in contrast to 12 and $5 \%$, respectively. The above facts may refer to some unique, specific, perhaps less studied features of the biosynthetic pathways of these organisms.

\section{The Numbers of (Existing and Possible) Microbial Metabolites \\ (How many bioactive microbial metabolites are known, indeed?)}

It may be an academic question, but it should be interesting as well, what is the (more or less) real number of the presently known microbial antibiotics? The number of the really known chemical entities, for various reasons, may be somewhat different than it is estimated on the simple survey of the literature. We has to take into account at least two specific points of view. Namely, the known, but 
neglected identities and the obscure origin of some plant and marine compounds.

\section{Problems of the Dereplication and the Nomenclature}

Hundreds of laboratories are engaged worldwide in the search for the discovery of new secondary metabolites by screening of ten thousands and sometimes millions of microbes. It is not surprising that during the course of this tremendous work the same metabolites have been discovered accidentally in the past (and perhaps in the present too) in multiple occasions. To avoid further confusing situations, the exact identification and correct naming of newly discovered metabolites have outstanding significance.

It is well known that in respect of the cost and time saving, the early evidence that an isolated (or detected) metabolite is a really new one or it may be identical with an already published compound, or in other words, the dereplication is one of the most essential element for all screening strategies. Unfortunately, these efforts frequently proved to be unsuccessful and in the scientific literature a large number of undesirable duplications have been occurring. For example in the early years of antibiotic era numerous compounds such as cinerubin, echinomycin, elaiophyllin, toyocamycin, etc. were discovered several times independently and 8 to 10 different names were given to these, in fact, identical compounds. Fortunately most of these duplicate names are no longer in common use, but only in the case of novobiocin was concluded a mutual agreement adopting a common name.

In the last decades over 1500 occasions already discovered compounds were isolated and published as new ones and were named, in fact, incorrectly. Later the detailed investigations (or structural elucidation) lead to the correct identification and these identities had been accepted and justified, but sometimes the situation remains for a long time confusing and some of these identical compounds are still appearing in the literature, unfortunately under different names.

Other problem, besides the unreliable dereplication, is the uncertain rules of the antibiotic nomenclature, the almost unregulated (ad hoc) situation in the naming of new antibiotics. First problem is that for about 1500 to 2000 published bioactive metabolites, even when they are exactly characterized pure compounds, no any name had been given. Sometimes for a compound only complicated chemical names or arbitrary given number or letter designations exist. This by itself makes communications difficult.

It is evident that in the scientific communications for any antibiotics the existence of only one, worldwide approved, simple, correct, euphonious (nonproprietary), generally accepted name, would be highly desirable. Unfortunately, the unambiguous chemical names in the case of complicated structures are inconvenient for general use. The worldwide adaptation of a single name for all substances/compounds would be significant achievement.

Scientists isolating a new compound naturally have indisputable right to give their own, distinctive name to the compound/s isolated, but the selection of an adequate name, based on some properties (chemical, biological properties or perhaps on biological origin) of the compound is expected. Independently from chosen whatever name, many confusing situations may be occurring:

- In the early years, for less well characterized antibiotic complexes, consisting of different proportion of the same components, usually totally different fantasy names were given. For example the practically identical streptothricin complexes are appearing under at least $30 \sim 40$, and the actinomycin $\mathrm{C}$ complex under about $10 \sim 15$ different names.

- For the isolated compounds, especially in the patent literature, frequently no specific name but only letter/number combinations are given. These terms sometimes became widely accepted, especially when they are used later in the scientific publications alike (FK-506), but very frequently, in the subsequent scientific publications these patent compounds appear under totally different names, sometimes without any cross-reference. (WF 1360/FR 900216=Rhizoxin; or TPU-025B = kosinostatin/quinocycline-B, etc.).

- For a compounds, which later proved to be identical with a known one, the name of the first isolated or best characterized compound is usually accepted. These common names may became general (picromycin, antimycin, etc.), but sometimes the other original names also remain in use and the same compounds appear consequently in different names in the literature. (Aureolic acid and Mithramycin; or in the Streptogramin family: Virginiamycin, Ostreogrycin, Pristinamycin, Staphylomycin, Mikamycin, Vernamycin, PA-114, etc.). In some cases, for various reasons the authors consequently use their own name, independently from the known identity, indicating (or not) the identity (Ristomycin=Ristocetin, Rubomycin-C $=$ Daunomycin) .

- In some specific examples when a practically important new compound proved to be identical with a formerly isolated but less well characterized old compound (usually with unknown structure), some legal problems may be arising: (Nancymycin=Rifamycin; Rugosin- $\mathrm{H}=$ Pyrrolnitrin; Ramycin=Fusidic acid; Ramiphyllin= 
Cyclosporin). The use of the name of the firstly discovered compound (which production is frequently unreproducable) usually disappear or became limited.

In fact, in the scientific literature for the microbial antibiotics, apart from the unnamed compounds, the chemical and Trade names, altogether 36000 different names, about twice as high as the number of the existing chemical compounds, occur. I know that the establishment a new general Antibiotic Nomenclature, or renovation of the rules as was proposed in 1965 at the Interscience Conference of Antimicrobial Agents and Chemotherapy (ICAAC), is almost impossible but to select and acknowledge a single internationally accepted general name for a chemical compound, representing a single antibiotic, seems to be realizable. The selection and adaptation of names meeting the necessary criteria certainly has strong interest in the scientific community.

\section{Hidden Identities}

I apologize in advance for the following cautious remarks, but over the above mentioned more or less recognized duplications, other obscure situations may also existing in the scientific literature. The detailed examination of data published, raised the possibility that in a few occasions, (notwithstanding the authors presumed most careful efforts), the accidental description of already published compounds as new ones, still occurs without revealing any reference to the possible identity or mentioning the close similarity. By a careful survey, using specific programs, I find more than one hundred examples, when the identity of two compounds, comparing the published physical, chemical, biological properties and the identical or similar producers, according to my best knowledge is more than probable. Sometimes the published structures are identical. In these cases I was unable to find, neither in the original nor in the subsequent publications any reference for the similarity or possible identity, but naturally these information may have evaded my attention. The literature data, of course, only indicate the supposed identity and undoubtedly further investigations or direct comparison can finally verify or exclude the identity. In Table 8 , illustrating the above statements, several examples of this hidden identities (with some concerning reference citations), are enlisted.

A longer list, more than hundred compound pairs (including the supposed identities) is available for request as a Supplementary Material from the Editorial Office of this Journal. To avoid the unnecessary duplications in the future, I think, both in Laboratories isolating new compounds and Editorial and Patent Offices accepting the
Table 8 The possible identical compounds* (Hidden identities)

\begin{tabular}{|c|c|c|}
\hline SCH-41956 (1) , ( $\alpha$-pyrone-I) (1a) & $=?$ & $\mathrm{BN}-213(\mathbf{2})$ \\
\hline Matlystatin A (3) & $=$ & BE-19093 (4) \\
\hline Dethymycin (5) & $=$ & Vancoresmyc \\
\hline Porothramycin (7) & $=$ & A-65636 (8) \\
\hline \multicolumn{3}{|c|}{ * Selection from a list of 102 compound pairs } \\
\hline \multicolumn{3}{|c|}{ 1) J Antibiot 55: 215 (2002); 1a) J Antibiot 56: 1033 (2003 } \\
\hline \multicolumn{3}{|c|}{ 2) Sci Rep, Meiji Seika K. N., 18: 7 (1979) } \\
\hline \multicolumn{3}{|c|}{ 3) J Antibiot 45: 1723 (1992); 47: 1481 (1994) } \\
\hline \multicolumn{3}{|c|}{ 4) Jpn Patent 1994: 25183, C. A., 121, 7449} \\
\hline \multicolumn{3}{|l|}{ 5) J Antibiot 45: 1819 (1992) } \\
\hline \multicolumn{3}{|l|}{ 6) Tetr Lett 43: 435: (2002) } \\
\hline \multicolumn{3}{|l|}{ 7) J Antibiot 41: 136 (1988) } \\
\hline \multicolumn{3}{|l|}{ 8) Abst ICAAC 27: 1004 (1987) } \\
\hline
\end{tabular}

publications, much more careful efforts are required to eliminate these inconvenient situations.

In summary, mainly as an unpleasant heritage of the past, in the scientific literature thousands of absolutely needless names and several "erroneous" compounds exist which may disturb the unmistakable communication. It is possible that there are perhaps more than hundred compounds occurring somewhat unjustifiably in the public and in the in-house databases, as well. The proved and potential identities, however, concern only a minor part (about 5\%) of our natural product library, but it indicates that the real number of the independent compounds may be lower by about several hundred than it is presently supposed.

\section{Obscure Origin of Compounds}

Over the above discussed possible duplications, there is an other aspect of this questionable topic. For totally different reason, the real numbers of microbial products may be somewhat higher as it presently seems. Namely, nobody knows today that how many microbial metabolites/antibiotics, are produced, in fact, by symbiotic microbes (bacteria, cyanobacteria, fungi) in marine invertebrates and by endophytic fungi living in the vascular plants, considering today as marine animal or plant products. By our days the opinion became generally accepted that at least in a part of the compounds isolated from marine invertebrates and in several cases from higher plants, the real producers are the symbiotic microbes; bacteria, cyanobacteria, algae or endophytic fungi. In several occasions (taxol, bryostatin, theopalauamide, caphalomannin, etc.) it had been exactly proved that symbiotic or endophytic microbes are responsible for the production of bioactive metabolites isolated from these 
"higher" organisms. The microbial origin of several marine derived compounds (tetrodotoxin, saxitoxin, swinholides, theopaulamide, bryostatins, etc.) already exactly documented.

I would like to mention only some simple facts supporting the reality of these opinions.

1. The chemical structures of several isolated "marine" or "plant" compounds (ansa-lactams, polyethers, polyines, scirpene lactones, unique heterocyclic or quinone systems) very frequently show surprising similarity to compounds isolated from microorganisms. Moreover, sometimes the same chemical compounds or its close analogues (toyocamycin, mimosamycin-renierone, some staurosporin, topsentin, nojirimycin analogs, etc.) are isolated besides the microbes (actinomycetales, cyanobacteria, fungi, etc.) from marine animals or higher plants, simultaneously.

2. There are over the individual compounds several specific chemical types for example cyclopolylactones, cyclopeptides, peptolides, diketopiperazines, indole derivatives, diphenyl-haloethers, unique heterocyclic systems, exemplified by ecteniascidins-saframycins/ safracins, maytansins-ansamitocins, verrucarinsbaccharins, okadaic acid/gonidomin-polyether antibiotics, prodigiosins-tambjamines, etc., which are widely distributed in the microbial world and are frequently occurring in compounds isolated from marine animals or higher plants, as well.

3. The symbiotic-associated microbial (bacterial/algal) population may consist of a great portion (sometimes $40 \sim 60 \%$ of the biomass) of the tissue of several marine animals. Virtually all higher plants, especially trees and shrubs, are host of microorganisms associated with different tissues of plant. The endophytic fungal population, are mainly unknown to the science, frequently produces defensive agents for their host and they are usually isolated and considered as "plant antibiotics".

4. The usually negligible or extremely low (e.g. $0.001 \sim 0.006 \%$ by weight) amount and variable yield of this types of bioactive compounds isolated from these animals or plants (e.g. $300 \mathrm{mg}$ halichondrin from one ton of sponges), also suggests their microbial origin.

5. Several microbes isolated from marine animals were able to produce the same compounds in laboratory, than was isolated formerly from their host.

It is true that due to the difficult isolation and cultivation of these symbiotic microbes, the number of direct proof is limited, but the reality of this imaginations is undoubted, only the dimension of this phenomenon is questionable. The problem is open and under extensive investigations worldwide.

It is fact that the real number of known chemical entities

on one hand are less and on the other are higher, but the above minor uncertainties do not touch the previous discussions and conclusions derived from the statistical data, at all.

Conclusions and Prospects

(How many microbial metabolites may be

discovered in the future?)

\section{Future Chances (Diversifying of Microbial Products)}

The total number of the microbial metabolites recognized until now, including both bioactive and inactive compounds, is around 50000 and the number of all known natural products is around one million. It is an obvious question, where is the border in the diversity of natural products? Where is the limit or is there any limit at all, in the continuous increase of the number of new microbial or natural compounds?

Regarding the possible number of bioactive natural products do not forget that natural product chemists in the past rarely investigated the isolated compounds, especially not for a wide range of bioactivity. Also remember that in the past countless of compounds, including microbial metabolites formerly believed to be inactive, proved to be active in later investigations, or were rediscovered with screening of different stock of microbes or with specific screening methods. It is unpredictable how many "new" bioactive metabolites will be discovered in this way.

The reinvestigation of the known natural/microbial products and especially the whole microbial population (natural products and microbiological libraries) with at wide variety, more selective, sensitive specific methods, especially in the light of the expanding knowledge of microbial genetics and the acquired knowledge about various genomes, would be especially fruitful. In all means, in the future we will discover more and more new functions, new activities of the microbial metabolites, will understand their real role and function and will expand the area of their practical utilization.

The general needs of the human society are continuously increasing. We need every new compounds which may be useful for the human society. More food, new drugs, and other goods are highly necessary for the benefit of humankind. The only question is the existence of sufficient natural and technical resources to fulfill these demands. Fortunately, in the area of the research of bioactive microbial products it seems that the ever expanding scientific and technical possibilities are increasing together with the continuously widening needs of the human 
therapy, veterinary and agriculture. The problem really is not whether we would be able to discover further new useful microbial compounds, but rather how can we optimize and quickly and effectively apply the chances derived from the new discoveries. How can we pick up and use effectively the proverbial needle found in the haystack.

According to my opinion our ability to discover new types of microbial products with new types of activities and new lead compounds for the drug (and agricultural product) development, may be limited only by three factors:

- the proper selection and certain luck to find and isolate the most promising new producers from our environment, to culture and maintain these producers, to modify the biosynthetic-genetic features of the strains isolated to obtain novel unnatural derivatives which cannot be obtained easily by chemical methods and finally the use of combinatorial chemical approaches to improve the natural leads, and

- the intellectual power in developing new, effective screening protocols; the proper selection of our targets; test microbes, biochemical-enzymological-pharmacological systems and host organisms, the adequate (selective, sensitive, automated) detection techniques including effective identification, and dereplication and isolation methods, and the efficient scale up and finally

- the effective data management, the ingenuity and cooperation of scientists from various disciplines.

\section{New Producers}

Natural resources (Maximizing the biodiversity)

Natural product resources, including the microbial world, are mainly unexplored both in its dimension and in the respect of geographic, ecological and environmental points of view. There surely exist, besides the presumed numbers of microorganisms, millions of microbes in the environment that are presently untouchable for the science. The DNA community analysis demonstrates the presence of much more novel organisms in the environmental samples, than those was originally believed, which are not detectable and cannot be isolated by "classical" methods.

If we compare the number of the known, described living species with the number of estimated and supposed species, may be existing in the nature, as summarized on Table 9, it can be seen that the biggest potential is in the fungal species. Fungi are the second largest group of eukaryotes next to the insects and exceed not only the bacteria and actinomycetes, but the higher plants also in terms of the number of possible existing species. It looks like that the word of fungi is one of the largest reservoir for isolating further bioactive metabolites.
There is great potency in the isolation and cultivation of microbes which are difficult to isolate or can not be isolated, less culturable or almost unculturable or simply slowly growing both in the groups of the well known or less explored types of microbes.

DNA analysis methods proved that the number of microbes in the soil is much higher than formerly thought. It was estimated that about $1 \%$ of the aquatic and $10 \%$ of the terrestrial microorganisms (perhaps only $0.1 \%$ of soil microbes), are readily cultured or "culturable" by conventional methods.

The application of new molecular techniques can greatly improve the effectivity of the exploration of uncommon, unreported groups of various microbes, first of all new actinomycetales strains, greatly increasing the chemical diversity.

Unique ecosystems as it is supposed, may result in unique organisms with unique metabolic pathways. The isolation of microbes from diverse ecosystems especially from under-represented sites (extreme circumstances, sea, etc.) may result in promising, new, until now unexplored producers. In general, the sea and to a lesser extent the rainforests are almost inexhaustible, untapped reservoirs for novel compounds. Besides the marine microorganisms, the soft-bodied marine animals and the endophytic fungi are living together with the green plants, represents important sources for new compounds. The isolation and cultivation of the aquatic, marine microorganisms (bacteria, fungi, actinomycetales), which are hard to maintain in laboratory environment, is an already widespread approach to increase the chemical diversity and it may give great impetus to the whole antibiotic research.

There is a big potential in the screening of the simply unknown species. The hardly detectable and almost unculturable actinomycetales species, their genetic exploitation and metabolic expression give also almost unlimited possibilities. The Cyanobacter and Myxobacter groups (not included specifically in Table 9) are also very promising as new sources of additional new bioactive compounds. Besides the known types, certainly exist additional new genera and phyla of microbes, in the nature. By genetic methods there is the possibility to obtain totally new phyla of microbes (Archaea, Archacebacteria).

\section{Genetic approaches (Genetic diversity)}

It is well known that small changes in culture conditions, or in the media, mixed- or co-cultivations, and similar simple methods all may result in big alteration in the biosynthesis of metabolites.

The cultivation of producers with unnatural precursors, the random mutagenesis and the use of genetically 
Table 9 The numbers of known and possible microbial (and other living) species

\begin{tabular}{lccc}
\hline Group & Described & Estimated/supposed & Deposited/producer \\
\hline Bacteria & $\sim 6000$ & $500000 / 1.5$ million & $3500 / 1000 \sim 1200$ \\
Actinomycetes & $\sim 4000$ & $35000 / 50000$ to 80000 & $3000 / 2000 \sim 2500$ \\
Fungi & $\sim 8000$ & 1.5 million/several millions & $15000 / 7000 \sim 8000$ \\
Viruses & $\sim 5000$ & $\sim 50000$ & $?$ \\
Algae & $\sim 2500$ & $\sim 50000 / \sim 400000$ & $\sim 2000 / 800 \sim 1000$ \\
& & & Investigated \\
Higher plants & $\sim 35000$ & 500000 to $600000 / 1.5$ million & $\sim 100000$ \\
Insects & Several millions/8 to 10 millions & $\sim 10000$ \\
Marine invertebrates & one million & 150000 to $200000 /$ several millions & 15000 to 20000 \\
Vertebrates & 20000 to 25000 & 50000 to 55000 & 20000 to 25000 \\
\hline
\end{tabular}

modified strains, are the simplest but promising opportunities to increase the chemical and biodiversity. The production of unnatural natural products, for example the preparation of penicillin $\mathrm{V}$ is the simplest long time known method.

The mutasynthesis or mutational biosynthesis, namely culturing microbes with unnatural precursors, also produced already hundreds of new hybrid molecules (aminoglycosides, peptides, etc.).

Microbes containing mutations in their biosynthetic genes can be fed by unnatural precursors, generating derivatives of complex natural products that are not easily obtainable by chemical methods. In the case of precursor directed biosynthesis when the producer is manipulated with "surgical" intervention to produce mainly the expected new hybrid compounds is also a promising approach.

Manipulations of microbial physiology, the combinatorial biosynthesis is an additional possibility. Molecular biological approaches allow the identification and activation of genes that operate the biosynthetic machinery of the microbes. Microbial genes can be engineered to produce hybrid enzymes that are capable to synthesizing a series of new analogous molecules (e.g. erythromycin derivatives). The cloning of biosynthetic pathway genes of e.g. Streptomyces can be transferred to other producers to help the discovery of new series of compounds. This combinatorial biosynthetic approach led e.g. to the direct fermentative production of epirubicin, one of the best antitumor anthracycline antibiotics, or to some fluorinated vancomycin derivatives.

In the genomes of microbes (actinomycetes, fungi, myxobacteria) there are encoded genes for numerous alternative biosynthetic pathways which allow the generation of new compounds. There are large number of silent genes responsible for production of various, perhaps new metabolites in actinomycetales e.g. in $S$. coelicolor A(3)2 [32]. The genome of $S$. avermitilis was found to contain over 30 gene clusters related to various metabolites [33], but so far only few ones such as avermectins, oligomycins and pentalenolactones have been isolated. The capacity of Streptomyces species to produce new types of secondary metabolites serving as new drug candidates seems to be almost inexhaustible.

The huge potential of genetic engineering, the application of molecular genetic techniques renders the hitherto uncultivable microorganisms accessible for generation of new secondary metabolites.

It seems to be the greatest advance, that in the future the genetic material derived from the microbes may be enough to generate new types of compounds without any complicated screening protocols. This recently emerged fascinating possibility is the direct access to the genetic information without isolation or cultivation of the fastidious microbes. This promising new approach represents the direct cloning of the metagenomic DNA obtained from digested unculturable microbes into an artificial vector which then transformed into a suitable culturable host (e.g. E. coli), and screening the resultant clone hoping to produce novel compounds.

In fact, the natural resources and their genetic manipulation are almost inexhaustible and certainly do not mean any limit in the increasing the number of further new "semi-natural" compounds. The combination of nucleic acid-sequencing techniques with molecular-phylogenetic analysis guarantees the increasing microbial diversity constituting an infinite pool of new potentially useful leads for the drugs and agrochemical compounds discovery. 


\section{Screening Protocols}

Targets (Maximizing the effectivity)

By our days the old problem of the screening processes, namely whether the investigation of only few producers exhaustively or the study of a great numbers of species using a single or few assays, or in other words, would it be better to treat a few strains in many ways or would it be advantageous to go after more isolates treat them a standard way, seems to be outdated. Availability of manpower is not a limiting factor anymore. We are nowadays able to investigate millions of producers in a short time by widely variable and diverse assay methods exhaustively, using a minimal manpower.

Besides the great technical improvements which had started already at the end of eighties, the anticipated widening of the possible targets available for automated screening methods is also tremendous. Advances in molecular and biochemical pharmacology have enabled the selection of specific molecular targets involved in the disease pathogenesis. The number of available cell-based, receptor binding, enzymatic and similar assay methods will yield ever increasing opportunities for further exploitation of the molecular diversity for treating and curing the most diverse human diseases. The progress of the human genome project and the advances of molecular biotechnology provide an explosion of new molecular targets implicated in human diseases, greatly increasing the range of assay methods available for screening projects.

The success of a screening depends on the application of suitable, sensitive, highly specific and effective, high throughput (automated, miniaturized) assay method. The automatic high throughput screening (HTS) techniques allow the screen of an almost unlimited number of samples in a very short time, working as fast as several minutes per sample, either 1000 to 5000 samples/day/robot The management of data generated, an integrated bioinformatics system to help the rapid selection of hits for candidate (lead) compounds, the integration of chemical fingerprinting (identification, correct dereplication) is naturally also highly required. In the future certainly new techniques and methods will be introduced. The extended use of the microtiter plates with high density spotting techniques, image analysis systems using biosensors, the nanotechnology and other new techniques may be ensure the maximization of these projects.

The increasing diversity of possible chemical structures in the result of these, advanced screening protocols, in all means will further increase the diversity of known biological activities and the usefulness of new compounds which will be discovered. In other words, the increasing biodiversity will deliver diversity of microbial products with increased chemical and mechanistic diversity. With the introduction of numerous new, very specific high throughput biochemical and pharmacological assay methods, based on the human genome, the number of the new targets and bioactivities will certainly further increase and the compounds discovered may be used both in the classical chemotherapy and in various "pharmacologicalphysiological" diseases as well as in different fields of agriculture.

Identification (Detection and dereplication)

The identification of active compounds from fermentation samples (extracts, whole broths) is one of the most complicated, labour intensive, time consuming step of the screening protocols. Recently the great progress in the robotic and automated methods in chromatographic isolation methods (LC-MS, LC-MS-ELSD, LC-NMR, HPLC-UV-VIS, HPLC-ELSD, HPLC-PDA (photodiode array detectors), HPCCC, CZE, etc.) and spectroscopic identification techniques (multi-dimensional NMR, X-Ray crystallography, NOESY, BMS, electrospray MS, HR-MS) are more or less satisfying the requirements of the modern HTS programs. For an effective dereplication and for selection the most promising new compounds a fully integrated bioinformatics system is the most critical factor. In the last decades using MS, NMR and X-Ray methods it became possible to solve the structure in a minimum quantities of natural products. The structure determination of a new compound, isolated perhaps only on $\mathrm{mg}$ scale is a relatively easy task.

Some aspects of this area, however, remain problematic e.g. in the case of highly water soluble and a practically insoluble compounds or those have low or no UV absorption.

The adequate quantities of compounds are critical, for detailed pharmacological and clinical tests but the Scale up and the production development is usually a simple task and can be critical only in the case of selected lead compounds.

\section{Data Management and Cooperation}

The evaluation of the mass of data generated in all steps of HTS methods is a more complicated task. It requires careful management across a range of disciplines. Integrated information systems, databases with suitable programs, the effective and critical analysis of all data represent the key area for the rapid delivery of new drug leads for the drug development. The voluminous data generated by the isolation and chemical identification 
techniques should be coupled via a direct data transfer with chemical and spectral libraries and natural product databases to identify the detected compounds and to eliminate the duplications. The success is greatly depending on the multidisciplinal development of sciences, biochemistry, microbiology, molecular biology, genetics, natural product and the general organic chemistry, robotics, informatics, microtechnical sciences.

Finally, we have to stress that the screening is not just a mechanistic routine work, besides the cooperation of scientist in all interested disciplines the human ingenuity and enthusiasm are the most important prerequisite. The close and effective cooperation of people working in this field is one of the most important factors of the success.

Summarizing our future chances it is clear that the expanding of economic sources of producers, the cultivation of unculturable, rare, fastidious, microorganisms, the new rational targets based on the new results of genetics and biotechnology, the HTS techniques with introduction of robotics technology and the extensive use of bioinformatics, made the screening more effective, less expensive and faster than ever. Simultaneously the anticipation that absolutely new types of new drugs will be discovered seems to be realistic. It is a great expectation that in the future by the expression of the (heterologous) genes from the unculturable microbes (the screening of the metagenom) may lead revolutionary new types of compounds with practically useful properties. But it is still the tale of the future.

\section{Epilogue}

Overviewing our present knowledge and the future perspectives it may be stated that there is no predictable limit in the further, perhaps not exponential but continuous growing of the number of new microbial metabolites, however, the qualitative improvement is much more important. Hopefully this increase will represent significant practical results both in the human therapy and agriculture. The tasks and possibilities are almost unlimited, only the economic and human effort invested, may be limiting factors. The greatest part of the world's biodiversity still unexplored and the new high-speed approaches allow its successful exploition. Cloning and genetic engineering offer alternative approaches and the chance of incorporating the suitable biosynthetic pathways from unculturable strains into appropriate hosts may be real. Natural products, in general, can be expected to play an important role in the ongoing transition from the empirical screening to the really rational drug design.
Myself I am cautiously optimistic for the future of the natural products research. We do not win the war against microbes, viruses and especially not against neoplastic and physiological diseases. The environmental issue will strengthen the research efforts in the area of agricultural compounds, and the war against human and animal diseases and for more productive and environmentally safe agriculture will continue for a long time, hopefully with good chances.

The philosophical discussion of various aspects of screening strategies, and the discussion of the open scientific, practical and economic problems is far from the primary aim and topic of this review, but unquestionable these questions are continuously in the foreground of the interest of the scientific community working in this area.

It has to say, I am somewhat anxious. Besides the tremendous technical improvements, achieved in the new screening projects, there are, however, some non-negligible practical, and economic problems:

Firstly. The problems of drug-target-host interactions. The hope that the new compounds derived from the HTS will fulfill the medical needs is constantly depending on three important requirements:

- the newly discovered agents will be specific enough to the molecular targets (pathogen microbes or mammalian organs), and

- the bioavailability (accessibility) of the molecular targets for the drug (uptake, transport) as well as

- the interaction of the newly discovered drug with the animal/human host. (toxic effect, pharmaco-kinetics)

A successful screening has to meet with these requirements. Without the proper answer to all of these questions there is no qualitative improvement. It seems the first requirements may satisfactorily be solved, but the fulfilling of the other two requirements, is definitely questionable. These are the areas where the genetic and combinatorial methods may be useful.

Second problem is the extreme overconcentration of the research, the overestimation of the new techniques and methodologies (as frequently happened in the past) and the hindering of some economic and human factors, may be alarming. Small research groups in all means have numerous burdensomes to carry out an effective HTS project, especially the in-house testing. The close industrial-academic cooperation or, as it happened already, the foundation of relatively small, specific biotechnological companies with sufficient scientific and economic background, may offer an effective avenue.

Third. Regarding to some specific economic points of view the increasing disparity is on the horizon between the rich-developed and the biodiversity-rich developing 
nations. The main natural resources (rainforest, unique environmental habitats, etc.) are mainly in the hand of developing nations, but the microbial screening in our days is absurd to carry out in these countries. Commercial rewards are usually not shared with the real source country. The trend, used to call as "biopiracy" is a difficult to solve problem. The low yield of plant and marine animal products will lead to increasing chemical and genetic approaches to produce these products by the advanced fermentative technologies also favorable only for rich nations.

Against to the above and some other pessimistic thoughts I am sure, that the essence of "the laws of applied microbiology" by the late David Perlman: "microorganisms can do or will do anything" is more valid than ever. My motto is: microorganisms (perhaps with a small help of the scientists) can do produce any compounds desired.

Acknowledgements I wish to express my appreciation to many colleagues in different parts of the world who helped me with various data and information and simply for sending me reprints of their hardly accessible papers. I am especially grateful to Satoshi Ōmura (Kitasato Institute), Arnold Demain (MIT), D. Hopwood (J. Innes Ctr.) and J. B. McAlpine (Ecopia) for their cooperation and helpful advices. I remember with a great gratitude to the late D. Perlman (Univ. Wisconsin), G. F. Gause (VNIIA), and M. Suffness (NCI, Bethesda) for their valuable advances in the past. I thank the contribution in the most different areas of our cooperation to A. Aszalos, M. Bostian, K. McNitt (at the NCI), R. Donovick (ATCC), M. Starkoff (CRC Press), and J. Buckingham (Chapman \& Hall). I wish to express my high gratitude to the late Profs. R. Bognár (University of Debrecen), and I. Horváth (IDR, Budapest), as well as to A. Simay (IVAX DRI) for their past and present helps. I would like to acknowledge the technical assistance to Gy. Máté (IVAX DRI).

\section{References}

1) Bérdy J, Magyar K. Antibiotics-A review. Process Biochem 3: 45-50 (1968)

2) Bérdy J. Recent developments of antibiotic research and classification of antibiotics according to chemical structure. Adv Appl Microbiol 18: 309-406 (1974)

3) Bostian M, McNitt K, Aszalos A, Bérdy J. Antibiotic identification: a computerized data base system. J Antibiot 30: 633-634 (1977)

4) Bérdy J. Recent advances in and prospects of antibiotic research. Process Biochem 15: 28-35 (1980)

5) Bérdy J. New ways to obtain new antibiotics. Chinese J Antibiot (Kangshengshu) 7: 348-360 (1984) (in English)

6) Bérdy J. Screening, classification and identification of microbial products. In Discovery and Isolation of Microbial
Products, Ed. Verral MS. Ellis Horwood, Chichester, pp. 9-31 (1985)

7) Bérdy J. New trends in the research of bioactive microbial metabolites. In Chemistry and Biotechnology of Biologically Active Natural Products, 4th Int. Conf., Budapest, 1987, pp. 269-291 (1988)

8) Bérdy J. Are actinomycetes exhausted as a source of secondary metabolites? In Biotehnologija (ISSN 0234-2758) Proc. 9th Int. Symp. On the Biology of Actinomycetes, Moscow 1994. Ed. Debanov VG. pp. 13-34 (1995)

9) Bérdy J. The discovery of new bioactive microbial metabolites: Screening and identification. In Bioactive Metabolites from Microorganisms. Ed. Bushell ME, Grafe U. Elsevier, Amsterdam (1989)

10) Bérdy J (Ed.), Aszalos A, Bostian M, McNitt K. Handbook of Antibiotic Compounds, CRC Press, Boca Raton (1980)

11) Dictionary of Natural Products. CRC Press, Boca Raton (2002)

12) Laatsch H. Antibase 2000, A Natural Products Database for Rapid Structure Determination, Chemical Concepts. Weinheim (2000)

13) Omura S. Splendid Gifts from Microorganisms. Kitasato Institute, Tokyo, pp. 1-344 (2003)

14) Knight V, Sanglier J-J, DiTullio D, Braccili S, Bonner P, Weaters J, Hughes D, Zhang L. Diversifying microbial natural products for drug discovery. Appl Microbiol Biotechnol 62: 446-458 (2003)

15) Donadio S, Carrano L, Brandi L, Serina S, Soffientini A, Raimondi E, Montanini N, Sosio M, Gualerzi CO. Targets and assays for discovering novel antibacterial agents. J Biotechnol 99: 175-185 (2002)

16) Donadio S, Monciardini P, Alduina R, Mazza P, Chiocchini C, Cavaletti L, Sosio M, Puglia AM. Microbial technologies for the discovery of novel bioactive metabolites. J Biotechnol 99: 187-198 (2002)

17) Proksch P, Edrata RA, Ebel R. Drugs from the seas - current status and microbiological implications. Appl Microbiol Biotechnol 59: 125-134 (2002)

18) Demain AL. Pharmacologically active secondary metabolites of microorganisms. Appl Microbiol Biotechnol 52: 455-463 (1999)

19) Farooq Biabani MA, Laatsch H. Advances in chemical studies on low-molecular weight metabolites of marine fungi. J Prakt Chem 340: 589-607 (1998)

20) Hill DC, Wrigley SK, Nisbet LJ. Novel screen methodologies for identification of new microbial metabolites with pharmacological activity. In Advances in Biochemical Engineering/Biotechnology, Vol. 59. Ed. Scheper T. Springer Verlag, Berlin, pp. 75-121 (1998)

21) Von Döhren H, Grafe U. General aspects of secondary metabolism. In Biotechnology, Vol. 7. Ed. Rehm H-J et al. VCH, Weinheim, pp. 1-55 (1997)

22) Porter N, Fox FM. Diversity of microbial productsDiscovery and application. Pesticide Sci 39: 161-168 (1993)

23) Yarbrough GG, Taylor DP, Rowlands RT, Crawford MS, 
Lasure LL. Screening microbial metabolites for new drugs - Theoretical and practical issues. J Antibiot 46: 535544 (1993)

24) Ōmura S. Trends in the search for bioactive microbial metabolites. J Industrial Microbiol 10: 135-156 (1992)

25) Demain AL, Fang A. The natural functions of secondary metabolites. In Advances in Biochemical Engineering/ Biotechnology, Vol. 69. Ed. Scheper T. Springer Verlag, Berlin, pp. 1-39 (2000)

26) Komada $T$, Sugiyama $Y$, Abe N, Imachi M, Hirota $H$, Koshino H, Hirota A. Revised structure of tetrapetalone A and its absolute stereochemistry. Tetrahedron Lett 44: 74177419 (2003)

27) Park H-R, Furihata K, Hayakawa Y, Shin-ya K. Versipelostatin, a novel GRP78/Bip molecular chaperone down-regulator of microbial origin. Tetrahedron Lett 43: 6941-6945 (2002)

28) Bérdy J, Kádár-Pauncz J, Méhesfalvi-Vajna Z, Horváth G, Gyimesi J. Metabolites of gentamicin producing Micromonospora species, Part 1. Isolation and identification of metabolites. J Antibiot 30: 945-953 (1977)

29) He H, Williamson RT, Shen B, Graziani EI, Yang HY, Sakya SM, Petersen PJ, Carter GT. Mannopeptimycins, novel antibacterial glycopeptides from Streptomyces hygroscopicus, LL-AC98. J Am Chem Soc 124: 9729-9736 (2002)
30) Sum P-E, How D, Torres N, Petersen PJ, Aschroft J, Grazianai EI, Koehn FE, Mansour TS. Synthesis and evaluation of ether and halogenated derivatives of mannopeptimycin glycopeptide antibiotics. Bioorg Med Chem Lett 13: 2505-2008 (2003)

31) Fehér M, Schmidt JM. Property distributions: Differences between drugs, natural products, and molecules from combinatorial chemistry. J Chem Inf Comput Sci 43: 218227 (2003)

32) Bentley SD, Chater KF, Cerdeno-Tarraga AM, Challis GL, Thomson NR, James KD, Harris DE, Quail MA, Kieser H, Harper D, Bateman A, Brown S, Chandra G, Chen CW, Collins M, Cronin A, Fraser A, Globe A, Hidalgo J, Hornsby T, Howarth S, Huang CH, Kieser T, Larke L, Murohy L, Oliver K, O’Neil S, Rabbinovitsch E, Rajandream MA, Rutherford K, Rutter S, Seeger K, Saunders D, Sharp S, Squares R, Squares S, Taylor K, Warren T, Wietzorrek A, Woodward J, Barrell BG, Parkhill J, Hopwood DA. Complete genome sequence of the model actinomycete Streptomyces coelicolor A3(2). Nature 417: 141-147 (2002)

33) Ikeda H, Ishikawa J, Hanamoto A, Shinose M, Kikuchi H, Shiba T, Sakaki Y, Hattori M, Omura S. Complete genome sequence and comparative analysis of the industrial microorganism Streptomyces avermitilis. Nature Biotechnol 21: 526-531 (2003) 